\title{
Coupling Finite Difference Methods and Integral Formulas for Elliptic Problems Arising in Fluid Mechanics
}

\author{
C. Albuquerque, G.-H. Cottet \\ Université Joseph Fourier Grenoble, LMC-IMAG, B. P. 53, \\ 38041 Grenoble Cedex 9, France
}

Received 2 April 2003; accepted 2 July 2003

Published online 00 Month 0000 in Wiley InterScience (www.interscience.wiley.com).

DOI 10.1002/num.10089

This article is devoted to the numerical analysis of two classes of iterative methods that combine integral formulas with finite-difference Poisson solvers for the solution of elliptic problems. The first method is in the spirit of the Schwarz domain decomposition method for exterior domains. The second one is motivated by potential calculations in free boundary problems and can be viewed as a numerical analytic continuation algorithm. Numerical tests are presented that confirm the convergence properties predicted by numerical analysis. ๑ 2004 Wiley Periodicals, Inc. Numer Methods Partial Differential Eq 20: 000-000, 2004

Keywords: elliptic problems; numerical analysis

\section{INTRODUCTION}

The numerical simulation of fluid flows frequently requires the solution of elliptic problems in complicated domains. Two examples that we will be considering here are exterior flows, where the fluid domain is unbounded, and free surface flows, where the fluid domain changes at each time step and may become very difficult to grid, either by finite differences or by finite elements.

In both cases integral equation methods may be used to handle far-field boundary conditions and/or to reduce the dimension of the problem. However these methods in general lead to linear systems that involve full matrices and may become time consuming.

The main idea behind the methods we consider here is to overcome these difficulties by combining, through an iterative procedure, integral formulas with finite difference discretizations of a modified domain with a simpler geometry. In the case of an exterior problem, the modified domain is a bounded subset of the original domain and the iterative method is reminiscent to a Schwarz domain decomposition technique. In the case of the free boundary problem, the modified domain is a fictitious domain that contains the fluid domain, and the

Correspondence to: G.-H. Cottet, Université Joseph Fourier Grenoble, LMC-IMAG, B. P. 53, 38041 Grenoble Cedex 9, France (e-mail: Georges-Henri.Cottet@imag.fr)

() 2004 Wiley Periodicals, Inc. 


\section{ALBUQUERQUE AND COTTET}

method can be viewed as a numerical analytic continuation algorithm. In either case the simplification in the discretized domains opens the possibility of using fast methods, resulting in a competitive way to solve the elliptic problems.

One motivation for coupling finite difference and integral equations for the simulation of Navier-Stokes equations in exterior domains is related to domain decomposition methods using different types of discretization in different subdomains. In a method proposed by Cottet [1], such a strategy was developed on the vorticity-velocity formulation of the Navier-Stokes equations to combine particle methods and finite difference methods where they are most appropriate. On the one hand particles are natural tools to describe wakes and can easily handle far-field boundary conditions; on the other hand finite difference methods are more flexible to implement no-slip boundary conditions on a solid boundary. The exterior domain is thus split into two domains: an annular domain around the solid boundary and an exterior domain.

To determine at each time step the velocity field associated to the vorticity field, a domain decomposition algorithm must then define interface velocity boundary conditions or, if the subdomains overlap, an iterative Schwarz method. In a particle method the velocity is computed from the vorticity by an integral formula - the Biot-Savart law-that requires potential at the boundary of the domain. In a straightforward application of the Schwarz method, these potentials should be computed through an integral equation. The method proposed in [1] (also used in [2]), and that we analyze in this article, bypasses this integral equation, and thus greatly simplifies the overall algorithm, by using potential values available in the finite difference domain.

In the numerical solution of water waves, the main difficulty is to discretize a domain with a boundary that may change at every time step. A discretization of the fluid domain (either by finite differences or by finite elements) is complex. However, if the flow is irrotational it can be described by the value of a potential at the free boundary. This potential has to be computed as the solution of an integral equation. The solution procedure becomes time consuming when the free boundary is complicated because more iterations are needed to obtain the convergence.

The method we consider here was proposed in [3] (see also [4]) and uses an eulerian grid for the fluid domain, extending the grid above the free boundary. The basic idea is to substitute the free boundary with a simpler artificial boundary above it and solve in the extended domain a problem whose solution approximates well the exact potential in the fluid domain. We obtain the boundary value at the artificial boundary by an iterative scheme that combines finite differences with an integral representation formula. The solution of the elliptic problem on the extended domain is easier and faster than in the fluid domain. The price we pay is that we have to solve not one but several elliptic problems in order to obtain our solution.

The existence of a condition on the artificial boundary that gives the exact potential on the fluid domain is only possible when there exists an analytic continuation of the potential from the fluid domain to the extended domain. However we are interested in a method to recover a good numerical approximation of the potential and its derivatives on the free boundary, even if an exact solution is not attainable. This can be obtained, provided that the artificial boundary is not far from the original one.

We remark that the algorithm can be viewed either as a general elliptic solver for complicated domains or as a numerical analytic continuation algorithm. This algorithm has been successfully applied to the numerical simulation of water waves $[4,5]$.

The objective of this article is to provide a numerical analysis of these two methods in several model cases as well as some numerical results. Applications to fluid mechanics problems can be found in $[1,2,4,5]$. 
The rest of the article is organized as follows. In section 2 we detail the integral formula/finite difference coupling approach for exterior problems. This leads to two methods, the convergence of which is analyzed in section 3. In section 4 we present numerical results that confirm the theoretical convergence results. In section 5 we briefly recall the mathematical formulation of the water waves problem and we state the analytic continuation algorithm for the calculation of the potential. In section 6 we study this algorithm for rectangular subdomains. In section 7 we describe a practical implementation of the analytic continuation algorithm in a general situation, having in mind its application to water waves simulation, and we present some numerical results confirming the convergence of the method. Finally we draw some conclusions in section 8 . The proof of three technical lemmas is postponed to the Appendix.

\section{SCHWARZ DOMAIN DECOMPOSITION FOR THE EXTERIOR POISSON PROBLEM: DEFINITIONS}

We start by considering a Poisson exterior problem with Dirichlet data and a far-field condition. Let $\Omega^{\prime}$ be a bounded domain of $\mathbb{R}^{2}$ with a smooth boundary $\Gamma$ and let $\Omega$ be the complementary of $\overline{\Omega^{\prime}}$ in $\mathbb{R}^{2}$. We want to approximate numerically the solution $u$ of

$$
\begin{cases}\Delta u=f & \text { in } \Omega \\ u=g & \text { on } \Gamma \\ u=c+O\left(\frac{1}{r}\right) & \text { when } r=\|x\| \rightarrow \infty\end{cases}
$$

for some constant $c \in \mathbb{R}$, under suitable conditions on $f$ and $g$. A possible approach to this problem may be an integral equation discretization (see, e.g., [6]).

Let us consider an overlapping domain decomposition for the case of an exterior domain. We decompose $\Omega$ in two subdomains $\Omega_{1}$ and $\Omega_{2}$, which overlap. The subdomain $\Omega_{1}$ is bounded with boundary $\Gamma \cup \Gamma_{1}$ and $\Omega_{2}$ is unbounded with boundary $\Gamma_{2}$ contained in $\Omega_{1}$. We also assume that $d\left(\Gamma_{1}, \Gamma_{2}\right)>0$ (see Fig. 1) and that the support of $f$ is contained in the complementary of $\overline{\Omega_{2}}$.

The classical Schwarz method leads to the following algorithm. We start with an arbitrary function $u_{0}$ in $\Gamma_{1}$ and, for $k \geq 1$, we define $u_{2 k-1}$ in $\Omega_{1}$ and $u_{2 k}$ in $\Omega_{2}$ by using the scheme

$$
\begin{cases}\Delta u_{2 k-1}=f & \text { in } \Omega_{1} \\ u_{2 k-1}=g & \text { on } \Gamma \\ u_{2 k-1}=u_{2 k-2} & \text { on } \Gamma_{1}\end{cases}
$$

and

$$
\begin{cases}\Delta u_{2 k}=0 & \text { in } \Omega_{2} \\ u_{2 k}=u_{2 k-1} & \text { on } \Gamma_{2} \\ u_{2 k}=c_{2 k}+O\left(\frac{1}{r}\right) & \text { when } r \rightarrow \infty\end{cases}
$$

with constants $c_{2 k} \in \mathbb{R}$. To simplify, we assume that the origin is in $\Omega^{\prime}$. This method will be hereafter denoted by method I. 


\section{ALBUQUERQUE AND COTTET}

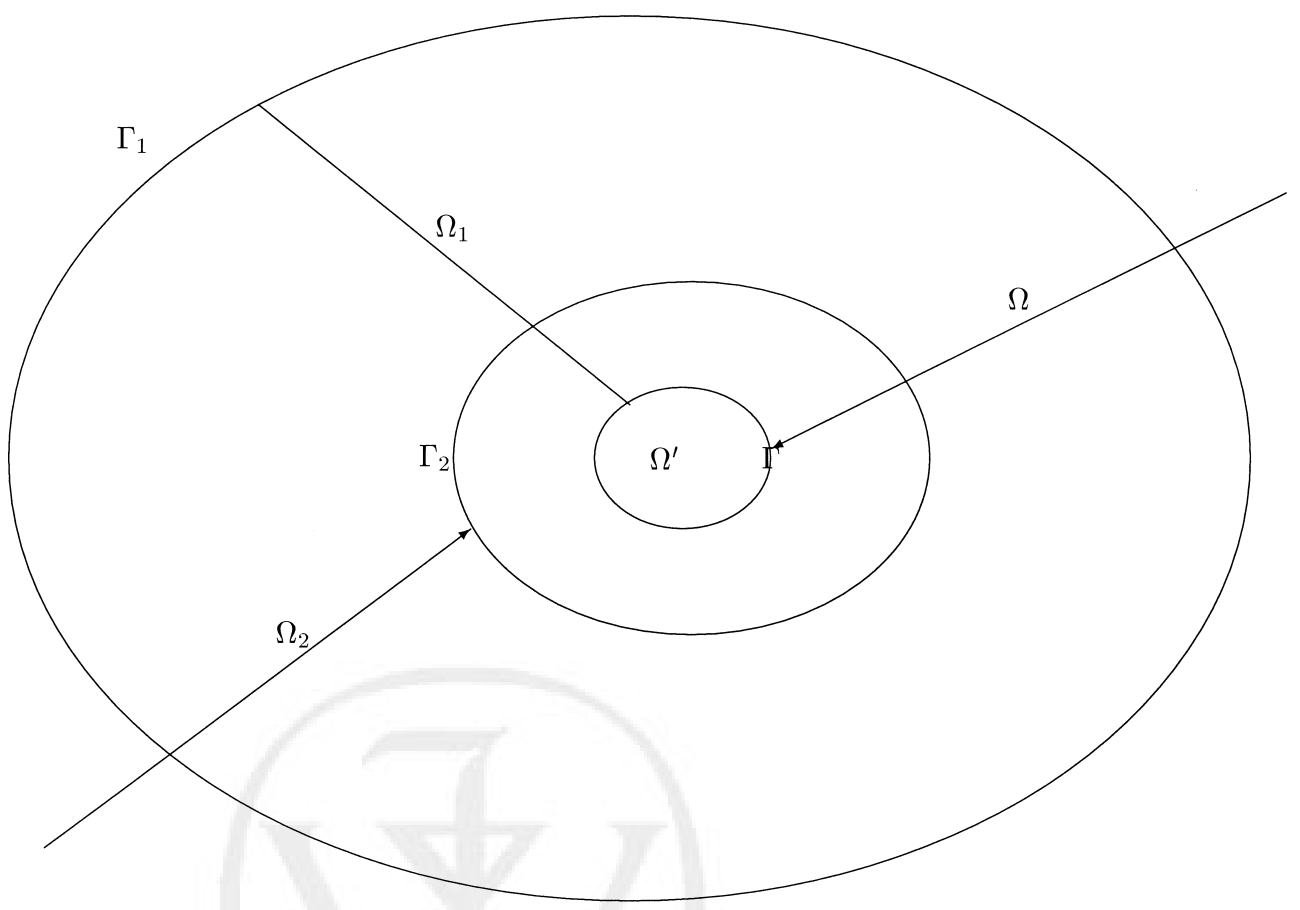

FIG. 1. Exterior domain decomposition.

Let us now come to an alternative version, originally proposed in [1], where the solution of (3) is approximated by integral formulas.

We know that, for each $k \geq 1$, the solution of (3) can be written as

$$
\begin{array}{r}
u_{2 k}(x)=\int_{\Gamma_{2}} u_{2 k-1}(y) \frac{\partial w}{\partial n}(y) d \gamma_{y}-\frac{1}{2 \pi} \int_{\Gamma_{2}} u_{2 k-1}(y) \frac{\partial}{\partial n_{y}} \log \|x-y\| d \gamma_{y} \\
+\frac{1}{2 \pi} \int_{\Gamma_{2}} \frac{\partial u_{2 k}}{\partial n}(y) \log \|x-y\| d \gamma_{y}
\end{array}
$$

where $w$ is the unique kernel solution [7] of

$$
\begin{cases}\Delta w=0 & \text { in } \Omega_{2} \\ w=0 & \text { on } \Gamma_{2} \\ \int_{\Gamma_{2}} \frac{\partial w}{\partial n} d \gamma=1 & \\ |w(x)|=O(\log \|x\|) & \text { when }\|x\| \rightarrow \infty \\ |\nabla w(x)|=O\left(\frac{1}{\|x\|}\right) & \text { when }\|x\| \rightarrow \infty\end{cases}
$$

We note that $w$ depends only on $\Gamma_{2}$. Equation (4) requires the solution of an integral equation on $\Gamma_{2}$. The modified Schwarz alternating method consists in replacing $\partial u_{2 k} / \partial n$ by $\partial u_{2 k-1} / \partial n$ in 
(4). Because we do not know the value of $\partial u_{2 k} / \partial n$ before solving (3), in formula (4) we replace it by $\partial u_{2 k-1} / \partial n$, as a natural guess deduced from the previous calculations in $\Omega$. However, it may occur that $\int_{\Gamma_{2}}\left(\partial u_{2 k-1} / \partial n\right) d \gamma \neq 0$ and in that case, because of the logarithmic kernel in the double layer integral, the approximation of $u_{2 k}$ grows at infinity as $O(\log r)$ instead of $O(1 / r)$. As $\Gamma_{1}$ may be far from $\Gamma_{2}$, the method may become unstable. Following [1], to correct this situation we substitute the integral formula (4) by an equivalent one, where the single layer integral

$$
\int_{\Gamma_{2}} \frac{\partial u_{2 k-1}}{\partial n}(y) \log \|x-y\| d \gamma_{y}
$$

is replaced by

$$
\int_{\Gamma_{2}} \frac{\partial u_{2 k-1}}{\partial n}(y) \log \left(\frac{\|x-y\|}{\|x\|}\right) d \gamma_{y}
$$

If we were using the exact value $\partial u_{2 k} / \partial n$, both formulas would be equivalent, because

$$
\int_{\Gamma_{2}} \frac{\partial u_{2 k}}{\partial n}(y) d \gamma_{y}=0
$$

The method we will consider is finally defined as follows. We start from an arbitrary $u_{0} \in$ $C\left(\Gamma_{1}\right)$; for $k \geq 1$ we define $u_{2 k-1}$ in $\Omega_{1}$ as the solution of

$$
\begin{cases}\Delta u_{2 k-1}=0 & \text { in } \Omega_{1} \\ u_{2 k-1}=g & \text { on } \Gamma \\ u_{2 k-1}=u_{2 k-2} & \text { on } \Gamma_{1}\end{cases}
$$

and we define $u_{2 k}$ in $\Omega_{2}$ by

$$
\begin{array}{r}
u_{2 k}(x)=\int_{\Gamma_{2}} u_{2 k-1}(y) \frac{\partial w}{\partial n}(y) d \gamma_{y}-\frac{1}{2 \pi} \int_{\Gamma_{2}} u_{2 k-1}(y) \frac{\partial}{\partial n_{y}} \log \|x-y\| d \gamma_{y} \\
+\frac{1}{2 \pi} \int_{\Gamma_{2}} \frac{\partial u_{2 k-1}}{\partial n}(y) \log \left(\frac{\|x-y\|}{\|x\|}\right) d \gamma_{y}
\end{array}
$$

where $w$ is the solution of (5). Equations (6) and (7) define a method hereafter denoted as method II.

If the subdomain overlapping region is large enough, we obtain uniform convergence of $u_{2 k}$ and $u_{2 k-1}$ to $u$, in $\Omega_{2} \backslash \Omega_{1}$ and $\Omega_{1}$, respectively. In fact, we prove a slightly stronger result.

Note that in the method proposed in [1], the iterative technique was combined with a finite difference solver in $\Omega_{1}$ and a particle discretization in $\Omega_{2}$, coupling finite differences and integral methods. In this article we only analyze the continuous underlying domain decompo- 


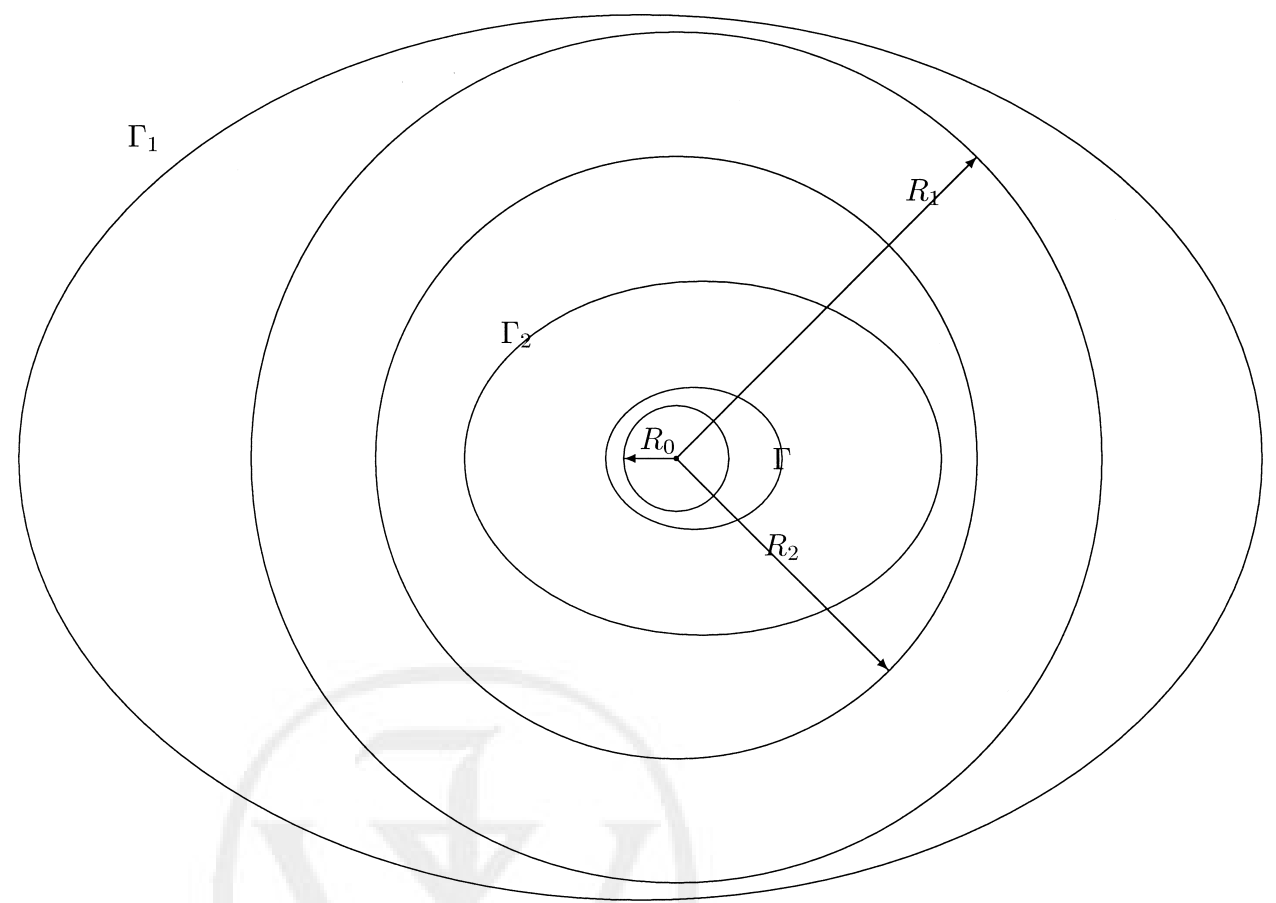

FIG. 2. The separation condition.

sition. However the numerical tests that we present in section 4 are based on a fully discretized algorithm.

\section{DOMAIN DECOMPOSITION ANALYSIS}

\subsection{Method I}

We start by studying the Schwarz method defined by (2)-(3). We assume that $g \in C(\Gamma)$ and $u_{0} \in C\left(\Gamma_{1}\right)$. We also make some geometrical assumptions on the subdomains of the decomposition. We consider $R_{0}$ such that the disk centered in the origin with radius $R_{0}$ is contained in $\Omega^{\prime}$. We also assume that we can separate $\Gamma_{2}$ and $\Gamma_{1}$ using two circumferences centered in the origin (see Fig. 2), i.e., there exist $R_{0}, R_{1}, R_{2}>0$ such that

$$
\begin{aligned}
& \left\{x \in \mathbb{R}^{2}:\|x\|<R_{0}\right\}=B\left(0, R_{0}\right) \subset \Omega^{\prime} \\
& \Gamma_{1} \subset \mathbb{R}^{2} \backslash B\left(0, R_{1}\right) \quad \Gamma_{2} \subset B\left(0, R_{2}\right) .
\end{aligned}
$$

Under these conditions we can prove the convergence of the domain decomposition method and estimate the rate of contraction of the error.

Theorem 1. If we choose $\Gamma_{1}$ such that $R_{1}$ is large enough compared to $R_{2}$ then, when $k \rightarrow \infty$, we have

$$
u_{2 k-1} \rightarrow u \quad \text { in } L^{\infty}\left(\Omega_{1}\right), \quad u_{2 k} \rightarrow u \quad \text { in } L^{\infty}\left(\Omega_{2}\right)
$$


Moreover, if we define, for $k \geq 1$,

$$
e_{2 k-1}=u-u_{2 k-1} \quad \text { in } \Omega_{1} \quad \text { and } \quad e_{2 k}=u-u_{2 k} \quad \text { in } \Omega_{2} \text {, }
$$

we have

$$
\left\|e_{2 k+1}\right\|_{\infty, \Omega_{1}} \leq \bar{c} \eta\left\|e_{2 k-1}\right\|_{\infty, \Omega_{1}} \quad\left\|e_{2 k+2}\right\|_{\infty, \Omega_{2}} \leq \bar{c} \eta\left\|e_{2 k}\right\|_{\infty, \Omega_{2}},
$$

with

$$
\eta \leq \frac{\log \left(R_{2}\right)-\log \left(R_{0}\right)}{\log \left(R_{1}\right)-\log \left(R_{0}\right)}
$$

and $\bar{c}$ depends only on $\Gamma_{2}$.

For the proof we need the following lemmas, proved in the Appendix.

Lemma 1. Let $h \in C\left(\Gamma_{1}\right)$ and $M>0$ be such that $|h(x)| \leq M$ for all $x \in \Gamma_{1}$, and let $v$ be the solution of

$$
\begin{cases}\Delta v=0 & \text { in } \Omega_{1} \\ v=0 & \text { on } \Gamma \\ v=h & \text { on } \Gamma_{1} .\end{cases}
$$

Then there exists $\eta, 0<\eta<1$, such that

$$
|v(x)| \leq \eta M, \quad \forall x \in \Gamma_{2}
$$

Furthermore $\eta$ depends only on the geometry and

$$
\eta \leq \frac{\log \left(R_{2}\right)-\log \left(R_{0}\right)}{\log \left(R_{1}\right)-\log \left(R_{0}\right)}
$$

Lemma 2. Let $h \in C\left(\Gamma_{2}\right)$ and $M>0$ be such that $|h(x)| \leq M$ for all $x \in \Gamma_{2}$ and let $v$ be the solution of

$$
\begin{cases}\Delta v=0 & \text { in } \Omega_{2} \\ v=h & \text { on } \Gamma_{2} \\ v=c+O\left(\frac{1}{r}\right) & \text { when } r=\|x\| \rightarrow \infty\end{cases}
$$

for some $c \in \mathbb{R}$. Then there exists a constant $\theta$, depending only on $\Gamma_{2}$, for which

$$
|v(x)| \leq \theta M, \quad \forall x \in \Omega_{2}
$$

Proof of Theorem 1. We start by considering $u_{1}$. We know that 


$$
\begin{cases}\Delta\left(u-u_{1}\right)=0 & \text { in } \Omega_{1} \\ u-u_{1}=0 & \text { on } \Gamma \\ u-u_{1}=u-u_{0} & \text { on } \Gamma_{1} .\end{cases}
$$

Let $L=\left\|u-u_{0}\right\|_{\infty, \Gamma_{1}}$. Then, by the weak maximum principle for harmonic functions, we have $\left\|u-u_{1}\right\|_{\infty, \Omega_{1}} \leq L$. By Lemma 1 there exists $\left.\eta \in\right] 0,1[$ such that

$$
\left\|u-u_{1}\right\|_{\infty, \Gamma_{2}} \leq \eta L
$$

and, by Lemma 2, there exists $\theta$ such that $\left\|u-u_{2}\right\|_{\infty, \Gamma_{1}} \leq \theta \eta L$ and, again by the weak maximum principle, $\left\|u-u_{3}\right\|_{\infty, \Omega_{1}} \leq \theta \eta L$. By induction we thus have $\left\|u-u_{2 k+1}\right\|_{\infty, \Omega_{1}} \leq(\theta \eta)^{k} L$. For the sequence $u_{2 k}$ we use Lemma 2 and (10) to obtain $\left\|u-u_{2}\right\|_{\infty, \Omega_{2}} \leq \theta \eta L$. Finally, we note that $\Gamma_{2}$ $\subset \Omega_{1}$, and we apply Lemma 2 to obtain

$$
\left\|u-u_{2 k}\right\|_{\infty, \Omega_{2}} \leq \theta\left\|u-u_{2 k-1}\right\|_{\infty, \Omega_{1}} \leq \theta(\theta \eta)^{k} L
$$

If we choose $\Gamma_{1}$ far enough from $\Gamma_{2}$, by Lemma 1 we can obtain $\eta$ as small as necessary to ensure that $\theta \eta<1$, and we obtain the geometrical convergence.

\subsection{Method II}

We now turn to the method defined by Equations (6) and (7). For this we need the following lemma.

Lemma 3. Let us assume that $\Omega$ is decomposed as in the hypotheses of Theorem 1. Moreover, we assume that $R_{1} \geq 2 R_{2}-R_{0}$. If $h \in C\left(\Gamma_{1}\right)$ is such that $|h(x)| \leq M$ in $\Gamma_{1}$ and $v \in C^{2}\left(\Omega_{1}\right) \cap$ $C\left(\overline{\Omega_{1}}\right)$ is the solution of

$$
\begin{cases}\Delta v=0 & \text { in } \Omega_{1} \\ v=0 & \text { on } \Gamma \\ v=h & \text { on } \Gamma_{1}\end{cases}
$$

then there exists $\eta^{*}>0$ such that

$$
\eta^{*} \leq \frac{\log \left(2 R_{2}-R_{0}\right)-\log \left(R_{0}\right)}{\log \left(R_{1}\right)-\log \left(R_{0}\right)}
$$

and, for all $x \in \Gamma_{2}$,

$$
\|\nabla v(x)\| \leq \frac{2 M \eta^{*}}{d\left(\Gamma_{2}, \Gamma\right)}
$$

The proof of this lemma is given in the Appendix.

We are now able to state and prove the convergence of the numerical method.

Theorem 2. We assume that $\Omega$ is decomposed as in Theorem 1 , with $R_{1} \geq 2 R_{2}-R_{0}$. Let $u$ be the solution of (1) with $g \in C(\Gamma)$. Let $u_{0}$ be an arbitrary function of $C\left(\Gamma_{1}\right)$. We consider 
$\left(u_{n}\right)_{n \in \mathbb{N}}$ defined by (6) and (7). If $\tilde{\Omega}$ is an open set such that $\tilde{\Omega} \subset \Omega_{2}$ with $d\left(\tilde{\Omega}, \Gamma_{2}\right)>0$, then, if $R_{1}$ is large enough, we have

$$
\begin{gathered}
u_{2 k-1} \rightarrow u \quad \text { in } L^{\infty}\left(\Omega_{1}\right) \\
u_{2 k} \rightarrow u \quad \text { in } L^{\infty}(\tilde{\Omega}) .
\end{gathered}
$$

Proof. For all $k \in \mathbb{N}$ we define

$$
\begin{gathered}
e_{2 k+1}=u-u_{2 k+1} \quad \text { in } \Omega_{1} \\
e_{2 k}=u-u_{2 k} \quad \text { in } \Omega_{2},
\end{gathered}
$$

and we know that $e_{2 k+1}$ is the solution of

$$
\begin{cases}\Delta e_{2 k+1}=0 & \text { in } \Omega_{1} \\ e_{2 k+1}=0 & \text { on } \Gamma \\ e_{2 k+1}=e_{2 k} & \text { on } \Gamma_{1}\end{cases}
$$

If we write $L=\max _{x \in \Gamma_{1}}\left|u(x)-u_{0}(x)\right|$, we have, by the weak maximum principle, $\left\|e_{1}\right\|_{\infty, \Omega_{1}} \leq$ $L$ and applying Lemma $1\left\|e_{1}\right\|_{\infty, \Gamma_{2}} \leq \eta L$. In general, for $k \geq 1$, we have

$$
\left\|e_{2 k+1}\right\|_{\infty, \Omega_{1}} \leq\left\|e_{2 k+1}\right\|_{\infty, \Gamma_{1}}=\left\|e_{2 k}\right\|_{\infty, \Gamma_{1}},
$$

and therefore

$$
\left\|e_{2 k+1}\right\|_{\infty, \Gamma_{2}} \leq \eta\left\|e_{2 k}\right\|_{\infty, \Gamma_{1}} .
$$

We now consider $\tilde{\Omega}$. To simplify we suppose that $\tilde{\Omega} \supset \mathbb{R}^{2} \backslash B\left(0, R_{1}\right)$. In what follows we will prove that we can find a positive constant $c$ (depending only on $R_{1}$ ) such that

$$
\left\|e_{2 k}\right\|_{\infty, \Gamma_{1}} \leq\left\|e_{2 k}\right\|_{\infty, \tilde{\Omega}} \leq c \cdot\left\|e_{2 k-2}\right\|_{\infty, \Gamma_{1}} \leq c \cdot\left\|e_{2 k-2}\right\|_{\infty, \tilde{\Omega}},
$$

and $c$ can be as small as we want, provided that $R_{1}$ is large enough. This proves (13) and, because $\Gamma_{1} \subset \widetilde{\Omega}$, we may combine this result with (14) to prove (12), thus concluding the proof of the theorem.

For $x \in \Omega_{2}$ (and, in particular, for $x \in \tilde{\Omega}$ ) we may write the integral representation formula for $u$ and, using the definition of $u_{2 k}$, we have

$$
\begin{array}{r}
e_{2 k}(x)=u(x)-u_{2 k}(x)=\int_{\Gamma_{2}} e_{2 k-1}(y) \frac{\partial w}{\partial n}(y) d \gamma_{y}-\frac{1}{2 \pi} \int_{\Gamma_{2}} e_{2 k-1}(y) \frac{\partial}{\partial n_{y}} \log \|x-y\| d \gamma_{y} \\
+\frac{1}{2 \pi} \int_{\Gamma_{2}} \frac{\partial e_{2 k-1}}{\partial n}(y) \log \left(\frac{\|x-y\|}{\|x\|}\right) d \gamma_{y},
\end{array}
$$




\section{ALBUQUERQUE AND COTTET}

and therefore

$$
\begin{array}{r}
\left|e_{2 k}(x)\right| \leq\left\|e_{2 k-1}\right\|_{\infty, \Gamma_{2}} \int_{\Gamma_{2}}\left|\frac{\partial w}{\partial n}(y)\right| d \gamma_{y}+\frac{1}{2 \pi}\left\|e_{2 k-1}\right\|_{\infty, \Gamma_{2}} \int_{\Gamma_{2}}\left|\frac{\partial}{\partial n_{y}} \log \|x-y\|\right| d \gamma_{y} \\
+\frac{1}{2 \pi}\left\|\frac{\partial e_{2 k-1}}{\partial n}\right\|_{\infty, \Gamma_{2}} \int_{\Gamma_{2}}\left|\log \left(\frac{\|x-y\|}{\|x\|}\right)\right| d \gamma_{y} .
\end{array}
$$

Applying Lemma 2 to $e_{2 k-1}$ and using (14), (15), we find that

$$
\begin{aligned}
\left|e_{2 k}(x)\right| \leq \eta\left\|e_{2 k-2}\right\|_{\infty, \Gamma_{1}} & \int_{\Gamma_{2}}\left|\frac{\partial w}{\partial n}(y)\right| d \gamma_{y}+\frac{\eta}{2 \pi}\left\|e_{2 k-2}\right\|_{\infty, \Gamma_{1}} \int_{\Gamma_{2}} \frac{1}{\|x-y\|} d \gamma_{y} \\
& +\frac{\eta^{*}}{\pi} \frac{\left\|e_{2 k-2}\right\|_{\infty, \Gamma_{1}}}{d\left(\Gamma_{2}, \Gamma\right)} \int_{\Gamma_{2}}\left|\log \left(\frac{\|x-y\|}{\|x\|}\right)\right| d \gamma_{y} \leq \max \left(\eta, \eta^{*}\right) \cdot M(x) \cdot\left\|e_{2 k-2}\right\|_{\infty, \Gamma_{1}},
\end{aligned}
$$

where

$$
\begin{aligned}
M(x)=\int_{\Gamma_{2}}\left|\frac{\partial w}{\partial n}(y)\right| d \gamma_{y} & +\frac{1}{2 \pi} \int_{\Gamma_{2}} \frac{1}{\|x-y\|} d \gamma_{y}+\frac{1}{\pi d\left(\Gamma_{2}, \Gamma\right)} \int_{\Gamma_{2}}\left|\log \left(\frac{\|x-y\|}{\|x\|}\right)\right| d \gamma_{y} \\
& \leq \int_{\Gamma_{2}}\left|\frac{\partial w}{\partial n}(y)\right| d \gamma_{y}+\frac{m\left(\Gamma_{2}\right)}{2 \pi d\left(\tilde{\Omega}, \Gamma_{2}\right)}+\frac{m\left(\Gamma_{2}\right) \cdot \bar{c}\left(d\left(\tilde{\Omega}, \Gamma_{2}\right), R_{2}, R_{0}\right)}{\pi d\left(\Gamma_{2}, \Gamma\right)}=\bar{M}
\end{aligned}
$$

Because $\eta$ and $\eta^{*}$ depend only on the geometry and decrease when $R_{1}$ increases, by placing $\Gamma_{1}$ far enough from $\Gamma_{2}$, we can make $\eta$ and $\eta^{*}$ small enough to have

$$
c=\max \left(\eta, \eta^{*}\right) \cdot \bar{M}<1
$$

thus proving the theorem.

\section{NUMERICAL TESTS}

In this section we describe the results of some numerical tests with the method presented in section 3.2. The geometry is the following:

$$
\Omega^{\prime}=B(0,1) \quad \Gamma_{2}=S(0,2) \quad \Gamma_{1}=S\left(0, R_{1}\right)
$$

with different values of $R_{1}$, to observe the error contraction as a function of $R_{1}$.

The interior problem is discretized using a fourth-order finite difference method over a polar grid. This regular grid has $m$ intervals in the radial direction and $n$ intervals in the angular direction. In the radial direction the number of intervals $m$ was chosen to produce $\Delta R=0.1$ and 
TABLE I. ARF (after five iterations) for tests with function $v$.

\begin{tabular}{rccc}
\hline$R_{1}$ & $\Delta R=0.1 \Delta \theta=\pi / 50$ & $\Delta R=0.05 \Delta \theta=\pi / 100$ & $\bar{\eta}$ \\
\hline 3.0 & 0.6304 & 0.6304 & 0.6309 \\
4.0 & 0.5001 & 0.5000 & 0.5000 \\
7.0 & 0.3567 & 0.3564 & 0.3562 \\
11.0 & 0.2901 & 0.2893 & 0.2891 \\
16.0 & 0.2519 & 0.2497 & 0.2500 \\
21.0 & 0.2304 & 0.2223 & 0.2277 \\
\hline
\end{tabular}

$\Delta R=0.05$. In the angular direction we used $n=100$ and $n=200$, thus yielding $\Delta \theta=\pi / 50$ and $\Delta \theta=\pi / 100$.

The line integrals are calculated using a composite trapezoid rule over $n$ points. At each iteration (6)-(7), we calculate the discrete- $L^{\infty}$ error of $u_{2 k-1}$ on $\Gamma_{2}$. From the second iteration on, we also calculate the error reduction factor $\left\|u-u_{2 k+1}\right\| /\left\|u-u_{2 k-1}\right\|$, where $u$ is the exact solution.

For tests, we choose $f$ and $g$ in (1) so that the exact solution is the harmonic function

$$
v(r, \theta)=\frac{\cos \theta}{r}
$$

Analogous results have been obtained with other functions of type $\left(\cos i \theta / r^{i}\right)(i \in \mathbb{N})$. We always start with $u_{0} \equiv 1$. For each level of grid resolution and after four iterations, we calculate the error average reduction factor (ARF). The ARF is the geometric mean of the error reduction factors from these four iterations. For each value of $R_{1}$, the numerical ARF is compared with the estimate from Theorem 1:

$$
\bar{\eta}=\frac{\log \left(R_{2}\right)-\log \left(R_{0}\right)}{\log \left(R_{1}\right)-\log \left(R_{0}\right)} .
$$

The results of the numerical tests are presented in Table I. The table shows that, when finite-differences and numerical integrations have reached convergence, the average reduction factor is almost exactly given by $\bar{\eta}$. Therefore, the estimate of the error contraction proved in

AQ: 1 Theorem 1 seems to apply as well to method II. This agreement suggests that the errors introduced in the practical algorithm by replacing $\partial u_{2 k} / \partial n$ with $\partial u_{2 k-1} / \partial n$ in (4) are not important for the numerical results.

When we increase the number of iterations, the reduction factor at each iteration deviates from the theoretical value, as the numerical errors in the finite-difference and quadrature approximation become predominant.

In Figs. 3 and 4, we also present plots of approximate and exact solutions. These plots were obtained after six iterations (6)-(7), with $v$ as data and using $R_{1}=7$ with $\Delta R=0.1$ and $\Delta \theta=$ $\pi / 50$.

\section{COUPLING TECHNIQUES FOR WATER-WAVES 5.1. Problem Setting}

We consider a two-dimensional inviscid, incompressible homogeneous fluid in a constant gravitational field, with a free boundary. We also assume that the flow is irrotational and 


\section{ALBUQUERQUE AND COTTET}

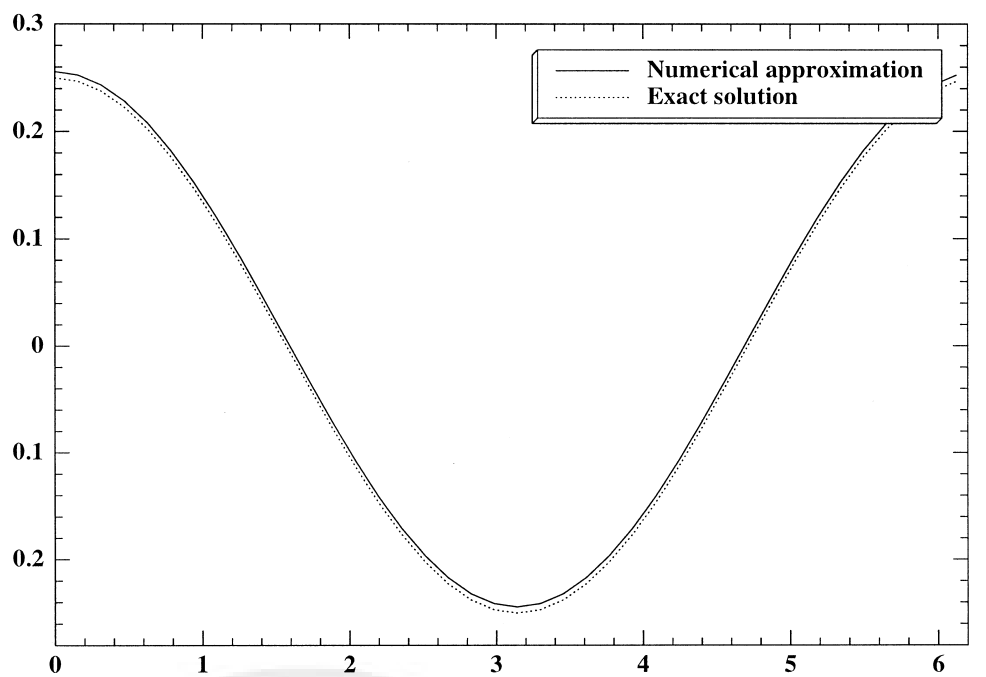

FIG. 3. Exact $(v)$ and approximate $\left(u_{12}\right)$ solutions on $\Gamma_{2}(r=2, \theta \in[0,2 \pi])$.

periodic in the horizontal direction, with period 1. No-through flow boundary condition is assumed at the bottom $\Gamma_{b}$ of the domain $\Omega$, which is located at $x_{2}=-b(b>0)$ (see Fig. 5).

The fluid is modeled by the incompressible Euler equations. The flow is characterized by the velocity field $\mathbf{v}=\left(v_{1}, v_{2}\right)$ and by the pressure $p$. The constant density is assumed to be 1 , for simplicity. The velocity field can be derived from a potential $\phi$ that is harmonic inside the fluid domain.

At the bottom of the fluid the no-through flow boundary condition gives, in terms of the potential function, an homogeneous Neumann boundary condition

$$
\frac{\partial \phi}{\partial n}=0 \quad \text { on } \Gamma_{b}
$$

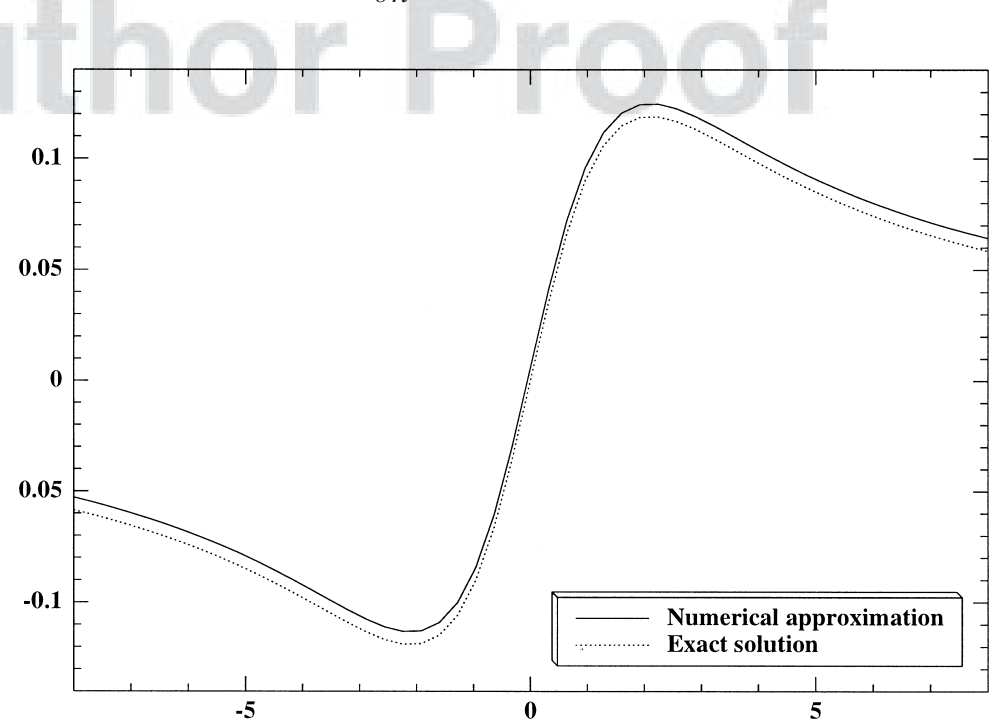

FIG. 4. Exact $(v)$ and approximate $\left(u_{12}\right)$ solutions on the line $y=2.1(x \in[-8,8])$. 


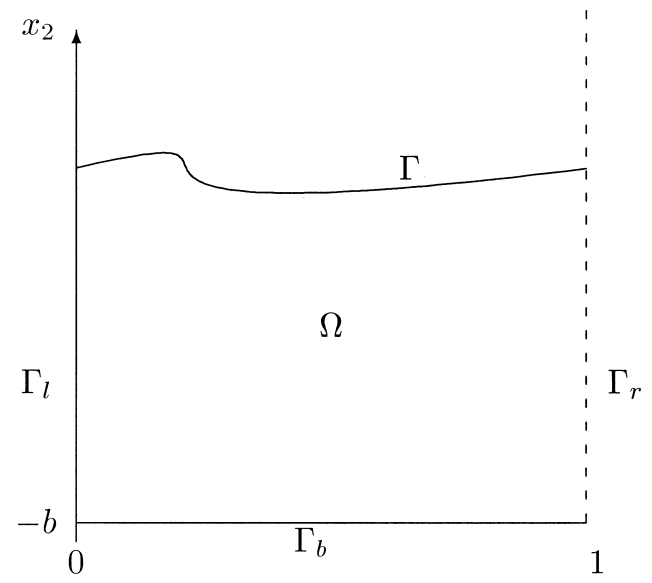

FIG. 5. Geometry of the problem.

For the evolution of the free surface we move each point with the local velocity of the fluid. We also need a dynamical boundary condition to determine the evolution of the potential on the free surface. Inside the fluid domain the potential function also satisfies the Bernoulli equation

$$
\frac{\partial \phi}{\partial t}+\frac{1}{2} \nabla \phi \cdot \nabla \phi+p+g x_{2}=0
$$

We assume that the pressure above the fluid is given. Using the continuity of the pressure through the free surface, Equation (17) holds on the free surface, with $p$ being the imposed pressure.

The complete system for the water-wave problem thus reads

$$
\begin{cases}\Delta \phi=0 & \text { in } \Omega \\ \frac{\partial \phi}{\partial n}=0 & \text { on } \Gamma_{b} \\ \frac{D \phi}{D t}=-p-g x_{2}+\frac{1}{2}|\nabla \phi|^{2} & \text { on } \Gamma \\ \text { periodic b. c. } & \end{cases}
$$

where $D \phi / D t$ is the total derivative. This system has to be completed by periodic boundary conditions in the horizontal direction (from now on periodicity will always be assumed).

For the numerical solution of problem (18) we have to couple at each time step through a boundary condition the solution of an elliptic problem with the lagrangian motion of the top boundary.

The main difficulty in the solution of the water waves problem lies in the repeated solution of elliptic problems where the free boundary $\Gamma$ changes at each time step and may become very complicated.

In the next section we describe the method used for the solution of these problems. 


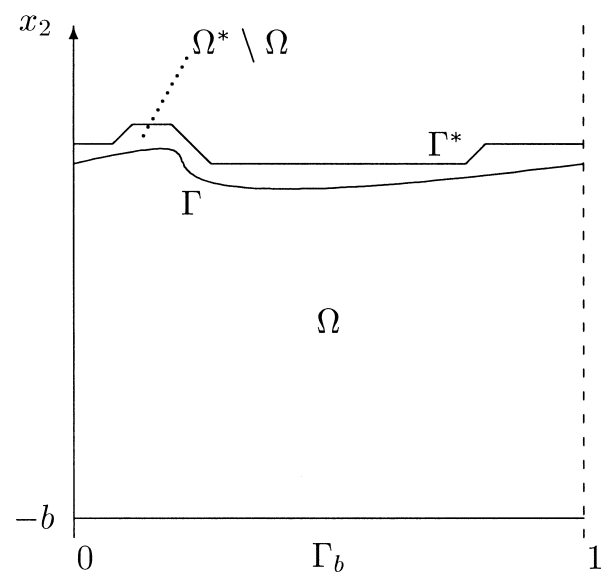

FIG. 6. Extended domain.

\subsection{The Analytic Continuation Algorithm}

Given a function $f$ on $\Gamma$, the potential is the solution of problem

$$
\begin{cases}\Delta \phi=0 & \text { in } \Omega \\ \phi=f & \text { on } \Gamma \\ \frac{\partial \phi}{\partial n}=0 & \text { on } \Gamma_{b}\end{cases}
$$

and we want to calculate $\nabla \phi$ on $\Gamma$. For that we will use an iterative method that constructs a function on an extended domain. This function approximates $\phi$ on the original domain.

We start by constructing an artificial boundary $\Gamma^{*}$ above the fluid free surface $\Gamma$ (see Fig. 6). The boundary $\Gamma^{*}$ is chosen in such a way that the extended domain allows an easy finitedifference discretization and is close to $\Gamma$. We denote the extended domain by $\Omega^{*}$.

If the potential $\phi$ on $\Omega$ had an analytic extension $\tilde{\phi}$ to $\Omega^{*}$, there would exist a boundary condition $\bar{\phi}=\tilde{\phi}_{\mid \Gamma^{*}}$ on $\Gamma^{*}$ such that the solution of the problem

$$
\begin{cases}\Delta \tilde{\phi}=0 & \text { in } \Omega^{*} \\ \tilde{\phi}=\bar{\phi} & \text { on } \Gamma^{*} \\ \frac{\partial \tilde{\phi}}{\partial n}=0 & \text { on } \Gamma_{b}\end{cases}
$$

would be equal to $\phi$ on $\Omega$.

If we do not have such boundary data $\bar{\phi}$ on $\Gamma^{*}$ (either because we do not know it or because there is no analytic extension of $\phi$ to $\Omega^{*}$ ), we will iterate from a tentative value $\phi^{p}$, in order to obtain an approximation of $\phi$ on $\Omega$. We start by solving problem (20), using $\phi^{p}$ instead of $\bar{\phi}$, and we obtain a function denoted by $\tilde{\phi}^{p}$ on $\Omega^{*}$. At this point $\tilde{\phi}_{\mid \Gamma}^{p}$ is probably different from $\phi_{\mid \Gamma}$. Now we would like to improve the approximation $\phi^{p}$ by using the information from $\phi_{\mid \Gamma}$. For this purpose we use a technique based on an integral representation formula. The basic idea is as follows. If we had the exact condition $\bar{\phi}$, then $\tilde{\phi}$ would be an harmonic function on $\Omega * \backslash \Omega$, and therefore we could represent its values on $\Gamma^{*}$ with an integral formula of the type: 


$$
\tilde{\phi}(x)=-\frac{2 \pi}{\theta}\left[\int_{\Gamma \cup \Gamma^{*}} \frac{\partial \tilde{\phi}}{\partial n}(y) G(x-y) d y-\int_{\Gamma \cup \Gamma^{*}} \tilde{\phi}(y) \frac{\partial G}{\partial n_{y}}(x-y) d y\right],
$$

where $G$ is the fundamental solution of the Laplace equation on a domain with periodic boundary conditions on the horizontal direction, $x$ is a point of $\Gamma^{*}$, and $\theta$ is the internal angle of $\Gamma^{*}$ at point $x$.

To calculate $\tilde{\phi}^{p+1}$ on $\Gamma^{*}$, we use formula (21) where boundary values are replaced on the one hand by values from $\tilde{\phi}^{p}$ for $\partial \tilde{\phi} / \partial n$ on $\Gamma \cup \Gamma^{*}$ and for $\tilde{\phi}$ on $\Gamma^{*}$, and on the other hand by the prescribed boundary value $f$ for $\tilde{\phi}$ on $\Gamma$.

The algorithm for the calculation of $\nabla \phi$ thus reads:

1. Start with some tentative value $\phi^{0}$ on $\Gamma^{*}$.

2. At iteration $p+1$, solve on the extended domain, the problem

$$
\begin{cases}\Delta \tilde{\phi}^{p}=0 & \text { in } \Omega^{*} \\ \tilde{\phi}^{p}=\phi^{p} & \text { on } \Gamma^{*} \\ \frac{\partial \tilde{\phi}^{p}}{\partial n}=0 & \text { on } \Gamma_{b} .\end{cases}
$$

3. Calculate $\partial \tilde{\phi}^{p} / \partial n$ on $\Gamma$ and $\Gamma^{*}$.

4. Recalculate the value of the potential on $\Gamma^{*}$ using the formula

$$
\begin{array}{r}
\phi^{p+1}(x)=-\frac{2 \pi}{\theta}\left[\int_{\Gamma \cup \Gamma^{*}} \frac{\partial \tilde{\phi}^{p}}{\partial n}(y) G(x-y) d y-\int_{\Gamma^{*}} \phi^{p}(y) \frac{\partial G}{\partial n_{y}}(x-y) d y\right. \\
\left.-\int_{\Gamma} f(y) \frac{\partial G}{\partial n_{y}}(x-y) d y\right],
\end{array}
$$

where $G$ is the fundamental solution of the Laplace operator with periodic conditions in the horizontal direction.

5. Go to step 2 and repeat until some stopping criterion is satisfied.

6. Calculate $\nabla \tilde{\phi}^{P}$ on $\Gamma$, where $P$ is the final iteration.

There is an important aspect of the iteration that we must remark at this point. If we fix $f$, the iteration $\phi^{p} \mapsto \phi^{p+1}$ is not linear (except in the case $f=0$ ). However, if we write the iteration as a function of both $\phi^{p}$ and $f$, we have a linear map. If $\phi^{p+1}=L\left(\phi^{p}, f\right)$, then it is easy to verify that $L\left(\phi_{1}^{p}+\phi_{2}^{p}, f_{1}+f_{2}\right)=L\left(\phi_{1}^{p}, f_{1}\right)+L\left(\phi_{2}^{p}, f_{2}\right)$ and $L\left(\lambda \phi^{p}, \lambda f\right)=\lambda L\left(\phi^{p}, f\right)$. This will be used to prove the independence of the iteration of the Fourier modes.

\section{CONVERGENCE ANALYSIS FOR THE ANALYTIC CONTINUATION ALGORITHM}

We consider the model case when $\Gamma$ and $\Gamma^{*}$ are horizontal lines (see Fig. 7). We assume that $\Gamma=\left\{x=\left(x_{1}, x_{2}\right): x_{2}=0\right\}$ and $\Gamma^{*}=\left\{x=\left(x_{1}, x_{2}\right): x_{2}=\delta\right\}$.

Let us first sketch the results proved in this section. We assume that there is an analytic continuation of $\phi$ on $\Omega^{*}$, denoted by $\tilde{\phi}$. When $\Gamma$ and $\Gamma^{*}$ are horizontal lines, the Fourier modes 


\section{ALBUQUERQUE AND COTTET}

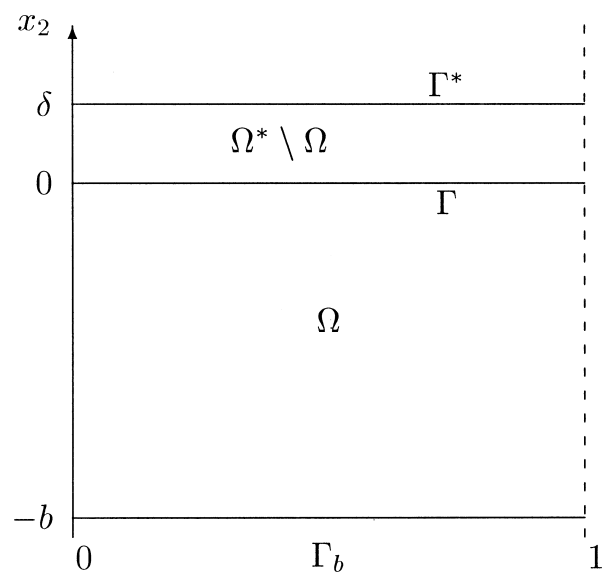

FIG. 7. Rectangular subdomains.

are decoupled. If both $f$ and $\phi^{0}$ have a finite number of Fourier modes, we will be able to analyze separately the Fourier modes in the iteration process. In particular if $f$ has only a finite number of Fourier modes, then the analytic continuation $\tilde{\phi}$ exists and we prove that $\phi^{p} \rightarrow \tilde{\phi}_{\mid \Gamma^{*}}$ and $\tilde{\phi}_{\mid \Gamma}^{p} \rightarrow f$ for any norm. Moreover, this convergence is geometrical. In particular,

$$
\phi^{p} \rightarrow \tilde{\phi}_{\mid \Gamma^{*}} \quad \text { in } H^{1 / 2}\left(\Gamma^{*}\right),
$$

and therefore,

$$
\tilde{\phi}^{p} \rightarrow \tilde{\phi} \quad \text { on } H^{1}\left(\Omega^{*}\right)
$$

If $f$ has an infinite number of Fourier modes, then the analytic continuation is only possible if the Fourier coefficients decay sufficiently fast. We are then able to prove similar convergence results. However, the convergence is geometrical if and only if $f$ has a finite number of Fourier modes. A remark on the practical implication of this fact is given at the end of this section.

In what follows we set $e^{p}=\phi^{p}-\tilde{\phi}_{\mid \Gamma^{*}}$ and $\tilde{e}^{p}=\tilde{\phi}^{p}-\tilde{\phi}$.

We start by considering the case when $f$ and $\phi^{p}$ have only the same Fourier mode, that is, $f=\bar{\mu}_{k} \cos \left(2 \pi k x_{1}\right)$ and $\phi^{p}=\lambda^{p} \cos \left(2 \pi k x_{1}\right)$.

Since $\tilde{\phi}$ is a harmonic function on $\Omega^{*}$, it can be written, for all $x \in \Gamma^{*}$,

$$
\tilde{\phi}(x)=-2\left[\int_{\Gamma \cup \Gamma^{*}} \frac{\partial \tilde{\phi}}{\partial n}(y) G(x-y) d y-\int_{\Gamma^{*}} \tilde{\phi}(y) \frac{\partial G}{\partial n_{y}}(x-y) d y-\int_{\Gamma} f(y) \frac{\partial G}{\partial n_{y}}(x-y) d y\right] .
$$

Upon subtracting from (23), one obtains

$$
e^{p+1}(x)=-2\left[\int_{\Gamma \cup \Gamma^{*}} \frac{\partial \tilde{e}^{p}}{\partial n}(y) G(x-y) d y-\int_{\Gamma^{*}} \tilde{e}^{p}(y) \frac{\partial G}{\partial n_{y}}(x-y) d y\right] .
$$


This is the integral representation of the function $\tilde{e}^{p}$ (harmonic in $\Omega$ ), except for the double-layer potential term on $\Gamma$. Therefore, we have, for all $x \in \Gamma^{*}$,

$$
e^{p+1}(x)=e^{p}(x)-2 \int_{\Gamma} \tilde{e}^{p}(y) \frac{\partial G}{\partial n_{y}}(x-y) d y .
$$

We then observe that the function

$$
\psi(x)=-2 \int_{\Gamma} \tilde{e}^{p}(y) \frac{\partial G}{\partial n_{y}}(x-y) d y
$$

is the unique periodic solution of the problem

$$
\left\{\begin{array}{l}
\Delta \psi=0 \quad \text { in }[0,1[\times \mathbb{R} \\
\psi \rightarrow-\int_{\Gamma} \tilde{e}^{p}(y) d y \quad \text { when } x_{2} \rightarrow+\infty \\
\psi \rightarrow \int_{\Gamma} \tilde{e}^{p}(y) d y \quad \text { when } x_{2} \rightarrow-\infty \\
{\left[\frac{\partial \psi}{\partial n}\right]_{\Gamma}=0} \\
{[\psi]_{\Gamma}=2 \tilde{e}^{p}}
\end{array}\right.
$$

where $[\cdot]$ is the jump on $\Gamma$

$$
[\gamma]_{\Gamma}=\gamma_{\Omega_{\mid \Gamma}}-\gamma_{\Omega * \backslash \Omega \mid \Gamma}
$$

Now $e^{p}=\alpha \cos \left(2 \pi k x_{1}\right)$ with $\alpha=\left(\lambda^{p}-\mu_{k}(\delta)\right)$ and it is easy to see that $\psi$ is given explicitly, for a value of $\beta$ to be determined, by the formula

$$
\psi(x)= \begin{cases}\beta \cos \left(2 \pi k x_{1}\right) e^{-2 \pi k x_{2}} & \text { for } x_{2}>0 \\ -\beta \cos \left(2 \pi k x_{1}\right) e^{-2 \pi k x_{2}} & \text { for } x_{2}<0\end{cases}
$$

and thus

$$
2 \tilde{e}^{p}\left(x_{1}, 0\right)=[\psi]_{\Gamma}\left(x_{1}\right)=-2 \beta \cos \left(2 \pi k x_{1}\right)
$$

We remark that $\psi$ has only the same Fourier mode as $f$ and $\phi^{p}$. By calculating explicitly $\tilde{e}^{p}$ as the solution of

$$
\begin{cases}\Delta \tilde{e}^{p}=0 & \text { in } \Omega^{*} \\ \tilde{e}^{p}=e^{p} & \text { on } \Gamma^{*} \\ \frac{\partial \tilde{e}^{p}}{\partial n}=0 & \text { on } \Gamma_{b}\end{cases}
$$


we can determine

$$
\beta=-\alpha \frac{\cosh (2 \pi k b)}{\cosh (2 \pi k(\delta+b))}
$$

this gives us $\psi$ in terms of $e^{p}$. Therefore we can write

$$
e^{p+1}=e^{p}+\psi=\alpha \cos \left(2 \pi k x_{1}\right)+\beta \cos \left(2 \pi k x_{1}\right) e^{-2 \pi k \delta}=\alpha c(k, \delta) \cos \left(2 \pi k x_{1}\right),
$$

where

$$
c(k, \delta)=1-\frac{\cosh (2 \pi k b) e^{-2 \pi k \delta}}{\cosh (2 \pi k(\delta+b))}
$$

Here we notice that $0<c(k, \delta)<1$ for all $k \in \mathbb{N}$ and all $\delta>0$. If $k=0$, then $c(0, \delta)=0$. The function $c(\cdot, \delta$ ) increases with $k$ (for a fixed $\delta$ ) and $c(k, \delta) \rightarrow 1$ as $k \rightarrow \infty, c(k, \delta) \rightarrow 1$ as $\delta \rightarrow \infty$ and $c(k, \delta) \rightarrow 0$ as $\delta \rightarrow 0$.

We are now able to analyze the evolution of the error in the iterative process. We have $e^{p+1}=c(k, \delta) e^{p}$, meaning that if $f$ and $\phi^{p}$ have only the same Fourier mode then $\phi^{p} \rightarrow \tilde{\phi}$ geometrically, in any norm. In the case $k=0$ ( $f$ and $\phi^{p}$ are both constant functions) the error becomes zero in only one iteration.

From the above properties of $c(k, \delta)$ we also conclude that, for a single Fourier mode, the iterations always converge. However, for higher Fourier modes $(k \rightarrow \infty)$ the convergence is slower. The distance $\delta$ between $\Gamma^{*}$ and $\Gamma$ has also an important effect on the rate of convergence. When $\Gamma^{*}$ is close to $\Gamma(\delta \rightarrow 0)$ the convergence becomes faster. In the application of the algorithm, $\Gamma^{*}$ must be chosen to be as close to $\Gamma$ as possible. However, the numerical stability of the calculation of the integral formula on $\Gamma$ imposes some restrictions to this proximity. We will come back to this issue in section 7 .

We now consider the case $f=\sum_{k=0}^{n} f_{k}$ with $f_{k}=\overline{\mu_{k}} \cos \left(2 \pi k x_{1}\right)$ and $\phi^{p}=\sum_{k=0}^{n} \phi_{k}^{p}$ with $\phi_{k}^{p}$ $=\lambda_{k}^{p} \cos \left(2 \pi k x_{1}\right)$. Using the operator $L$ defined in subsection 5.2, we get

$$
\phi^{p+1}=L\left(\phi^{p}, f\right)=L\left(\sum_{k=0}^{n} \phi_{k}^{p}, \sum_{k=0}^{n} f_{k}\right)=\sum_{k=0}^{n} L\left(\phi_{k}^{p}, f_{k}\right)=\sum_{k=0}^{n} \phi_{k}^{p+1}
$$

and $\phi_{k}^{p+1}$ is dependent only on $\phi_{p}^{k}$ and $f_{k}$; the Fourier modes iterate independently and we can superpose the results from the single Fourier mode case:

$$
\begin{gathered}
\lambda_{k}^{p} \rightarrow \mu_{k}(\delta) \quad \text { when } k \rightarrow \infty \\
\lambda_{k}^{p+1}-\mu_{k}(\delta)=c(k, \delta)\left[\lambda_{k}^{p}-\mu_{k}(\delta)\right] \\
\gamma_{k}^{p+1}-\nu_{k}(\delta)=c(k, \delta)\left[\gamma_{k}^{p}-\nu_{k}(\delta)\right],
\end{gathered}
$$

$\mu_{k}(\delta)$ and $\nu_{k}(\delta)$ being the exact values. Therefore, if both $f$ and $\phi^{p}$ have a finite number of Fourier modes, then the convergence is geometrical, with rate dependent on the largest $k$. 
We finally consider the case of analytic data $f$ with an infinite number of Fourier modes. We assume that there exists $\tilde{\phi}$, an analytic continuation of $\phi$. In this case we also obtain the convergence of $\phi^{p}$ in $H^{1 / 2}\left(\Gamma^{*}\right)$; however, we prove that the convergence cannot be geometrical in $H^{1 / 2}\left(\Gamma^{*}\right)$; even if we consider the weaker norm of $L^{2}\left(\Gamma^{*}\right)$, we still do not have geometrical convergence.

We start by considering the functional framework for the study of the convergence of the iterations. Because the set of functions $\left\{1, \cos \left(2 \pi k x_{1}\right), \sin \left(2 \pi k x_{1}\right)\right\}_{k \in \mathbb{N}}$ is a Hilbert base of $H^{1 / 2}(\Gamma)$ and $H^{1 / 2}\left(\Gamma^{*}\right), f$ takes the form

$$
f=\bar{\mu}_{0}+\sum_{k=1}^{\infty} \bar{\mu}_{k} \cos \left(2 \pi k x_{1}\right)+\bar{\nu}_{k} \sin \left(2 \pi k x_{1}\right),
$$

with

$$
\sum_{k=1}^{\infty} k\left(\bar{\mu}_{k}^{2}+\bar{\nu}_{k}^{2}\right)<+\infty
$$

Then, if an analytic continuation $\tilde{\phi}$ exists, it will take the form

$$
\tilde{\phi}_{\mid \Gamma^{*}}=\mu_{0}(\delta)+\sum_{k=1}^{\infty} \mu_{k}(\delta) \cos \left(2 \pi k x_{1}\right)+\nu_{k}(\delta) \sin \left(2 \pi k x_{1}\right),
$$

and the coefficients must verify

$$
\sum_{k=1}^{\infty} k\left(\mu_{k}(\delta)^{2}+\nu_{k}(\delta)^{2}\right)<+\infty
$$

As $\mu_{k}(\delta) \sim e^{4 \pi k \delta} \bar{\mu}_{k}$ and $\nu_{k}(\delta) \sim e^{4 \pi k \delta} \bar{\nu}_{k}$, the coefficients of $f$ must also satisfy

$$
\sum_{k=1}^{\infty} k e^{8 \pi k \delta}\left(\bar{\mu}_{k}^{2}+\bar{\nu}_{k}^{2}\right)<+\infty
$$

This means that the coefficients of $f$ must decay exponentially with $k$. The exponential decay corresponds to an analytic function. This is reasonable because the restriction to $\Gamma$ of a function that is harmonic in $\Omega^{*}$ must be analytic on $\Gamma$.

For numerical purposes, let us point out that we can always replace $f$ by a close enough finite sum of trigonometric polynomials. This approximation can be chosen as close to $f$ as we wish, in the $H^{1 / 2}(\Gamma)$ norm, and from that we solve our initial problem to any desired accuracy.

If $f$ has an infinite number of Fourier modes and $\phi$ admits an extension $\tilde{\phi}$ to $\Omega^{*}$, then $e^{p}$ takes the form 


$$
e^{p}=\left[\lambda_{0}^{p}-\mu_{0}(\delta)\right]+\sum_{k=1}^{\infty}\left[\lambda_{k}^{p}-\mu_{k}(\delta)\right] \cos \left(2 \pi k x_{1}\right)+\left[\gamma_{k}^{p}-\nu_{k}(\delta)\right] \sin \left(2 \pi k x_{1}\right)
$$

and

$$
e^{p+1}=\left[\lambda_{0}^{p+1}-\mu_{0}(\delta)\right]+\sum_{k=1}^{\infty}\left[\lambda_{k}^{p+1}-\mu_{k}(\delta)\right] \cos \left(2 \pi k x_{1}\right)+\left[\gamma_{k}^{p+1}-\nu_{k}(\delta)\right] \sin \left(2 \pi k x_{1}\right)
$$

Because the Fourier modes iterate independently, we know that $\lambda_{k}^{p+1}$ is dependent only on $\lambda_{k}^{p}$ and $\bar{\mu}_{k}$. Similarly $\gamma_{k}^{p+1}$ is dependent only on $\gamma_{k}^{p}$ and $\bar{\nu}_{k}$. Moreover, we can use relations (25) and (26). We are now able to prove the convergence results.

Theorem 3. Assume $f \in H^{1 / 2}(\Gamma)$ is such that the solution $\phi$ of (19) has an analytic extension $\tilde{\phi}$ to $\Omega^{*}$. Then, for any $\phi^{0} \in H^{1 / 2}\left(\Gamma^{*}\right)$, the sequence $\phi^{p}$ defined in subsection 5.2 converges to $\tilde{\phi}_{\mid \Gamma^{*}}$ in $H^{1 / 2}\left(\Gamma^{*}\right)$. Moreover, if $\phi^{0}=0$ then the convergence is geometrical if and only if $f$ has a finite number of Fourier components.

The proof that the convergence cannot be geometrical is obtained using a contradiction argument. If there were some rate of contraction $0 \leq \rho<1$ such that $\left\|e^{p+1}\right\| \leq \rho\left\|e^{p}\right\|$, we could separate a finite number of Fourier modes with an error contraction rate $c(k, \delta) \leq \rho$ and an infinite number of Fourier modes for which $c(k, \delta)>\rho$. When the number of iterations increases, the first part of the error decays faster than the second. After a sufficient number of iterations, the Fourier modes for which $c(k, \delta)>\rho$ holds become important enough, and we have $\left\|e^{p+1}\right\|>\rho\left\|e^{p}\right\|$, which contradicts the initial assumption.

More precisely we can state the following proof.

Proof of Theorem 3. We have

$$
\left\|e^{p}\right\|_{H^{1 / 2}\left(\Gamma^{*}\right)}=\left(\left|\lambda_{0}^{p}-\mu_{0}(\delta)\right|^{2}+\sum_{k=1}^{\infty} k\left[\left(\lambda_{k}^{p}-\mu_{k}(\delta)\right)^{2}+\left(\gamma_{k}^{p}-\nu_{k}(\delta)\right)^{2}\right]\right)^{1 / 2}
$$

and

$$
\left\|e^{p+1}\right\|_{H^{1 / 2}\left(\Gamma^{*}\right)}=\left(\left|\lambda_{0}^{p+1}-\mu_{0}(\delta)\right|^{2}+\sum_{k=1}^{\infty} k\left[\left(\lambda_{k}^{p+1}-\mu_{k}(\delta)\right)^{2}+\left(\gamma_{k}^{p+1}-\nu_{k}(\delta)\right)^{2}\right]\right)^{1 / 2}
$$

Using (25) and (26) it is clear that we always have

$$
\left\|e^{p+1}\right\|_{H^{1 / 2}\left(\Gamma^{*}\right)} \leq\left\|e^{p}\right\|_{H^{1 / 2}\left(\Gamma^{*}\right)} .
$$

Furthermore, if $e^{p}$ has a finite number of Fourier components (which is the case when both $f$ and $\phi^{p}$ have a finite number of Fourier components), we have

$$
\left\|e^{p+1}\right\|_{H^{1 / 2}\left(\Gamma^{*}\right)} \leq c(N, \delta)\left\|e^{p}\right\|_{H^{1 / 2}\left(\Gamma^{*}\right)}
$$

for some natural $N$. 
If $e^{p}$ has an infinite number of Fourier components, then for any positive $\epsilon$, we can write

$$
e^{p}=e_{*}^{p}+e_{* *}^{p},
$$

with

$$
e_{*}^{p}=\left[\lambda_{0}^{p}-\mu_{0}(\delta)\right]+\sum_{k=1}^{M}\left[\lambda_{k}^{p}-\mu_{k}(\delta)\right] \cos \left(2 \pi k x_{1}\right)+\left[\gamma_{k}^{p}-\nu_{k}(\delta)\right] \sin \left(2 \pi k x_{1}\right),
$$

and

$$
e_{* *}^{p}=\sum_{k=M+1}^{\infty}\left[\lambda_{k}^{p}-\mu_{k}(\delta)\right] \cos \left(2 \pi k x_{1}\right)+\left[\gamma_{k}^{p}-\nu_{k}(\delta)\right] \sin \left(2 \pi k x_{1}\right)
$$

and where $M$ is chosen large enough to ensure that

$$
\left\|e_{* *}^{p}\right\|_{H^{1 / 2}\left(\Gamma^{*}\right)}<\frac{\epsilon}{\sqrt{2}}
$$

We also know [from (25) and (26)] that

$$
\left\|e_{*}^{p+1}\right\|_{H^{1 / 2}\left(\Gamma^{*}\right)}<c(M, \delta)\left\|e_{*}^{p}\right\|_{H^{1 / 2}\left(\Gamma^{*}\right)} .
$$

Calculating $q$ iterates, we get

$$
e^{p+q}=e_{*}^{p+q}+e_{* *}^{p+q}
$$

and

$$
\left\|e_{* *}^{q+q}\right\|_{H^{1 / 2}\left(\Gamma^{*}\right)}^{2} \leq\left\|e_{* *}^{p}\right\|_{H^{1 / 2}\left(\Gamma^{*}\right)}^{2}<\frac{\epsilon^{2}}{2}
$$

and

$$
\left\|e_{*}^{p+q}\right\|_{H^{1 / 2}\left(\Gamma^{*}\right)}^{2} \leq[c(M, \delta)]^{2 q}\left\|e_{*}^{p}\right\|_{H^{1 / 2}\left(\Gamma^{*}\right)}^{2} .
$$

As $0<c(M, \delta)<1$, if the number of iterations $q$ is sufficiently large, we have

$$
\left\|e_{*}^{p+q}\right\|_{H^{1 / 2}\left(\Gamma^{*}\right)}^{2}<\frac{\epsilon^{2}}{2}
$$

and therefore 


\section{ALBUQUERQUE AND COTTET}

$$
\left\|e^{p+q}\right\|_{H^{1 / 2}\left(\Gamma^{*}\right)}=\sqrt{\left\|e_{*}^{p+q}\right\|_{H^{1 / 2}\left(\Gamma^{*}\right)}^{2}+\left\|e_{* *}^{p+q}\right\|_{H^{1 / 2}\left(\Gamma^{*}\right)}^{2}}<\epsilon
$$

Because $\epsilon$ is an arbitrary positive number, we have proved that

$$
e^{p} \rightarrow 0 \quad \text { in } H^{1 / 2}\left(\Gamma^{*}\right),
$$

which means

$$
\phi^{p} \rightarrow \tilde{\phi}_{\mid \Gamma^{*}} \quad \text { in } H^{1 / 2}\left(\Gamma^{*}\right)
$$

To prove that this convergence cannot occur with a geometrically decreasing error, we assume, for simplicity, that

$$
e^{p}=\sum_{k=0}^{\infty} \xi_{k}^{p} \cos \left(2 \pi k x_{1}\right)
$$

with an infinite number of nonzero coefficients $\xi_{k}^{p}$.

In order to obtain a contradiction we also assume that there exists a number $\rho$ such that $0<$ $\rho<1$ and

$$
\left\|e^{p+1}\right\|_{H^{1 / 2}\left(\Gamma^{*}\right)} \leq \rho\left\|e^{p}\right\|_{H^{1 / 2}\left(\Gamma^{*}\right)} .
$$

Let $k_{0}$ be the smallest integer such that

$$
c\left(k_{0}, \delta\right)>\rho \quad \text { and } \quad \xi_{k_{0}}^{p} \neq 0
$$

We define the positive quotients

$$
\eta_{k}^{p}=\frac{\left|\xi_{k}^{p}\right|}{\left|\xi_{k_{0}}^{p}\right|}
$$

and we notice that, for $k<k_{0}$,

$$
\eta_{k}^{p} \rightarrow 0 \quad \text { when } \quad p \rightarrow \infty
$$

because either $\eta_{k}^{p}=0$ for all $p$ or $\eta_{k}^{p} \neq 0$ for all $p$, and in this case

$$
\frac{\eta_{k}^{p+1}}{\eta_{k}^{p}}=\frac{\left|\xi_{k}^{p+1}\right|}{\left|\xi_{k}^{p}\right|} \frac{\left|\xi_{k_{0}}^{p}\right|}{\left|\xi_{k_{0}}^{p+1}\right|}=\frac{c(k, \delta)}{c\left(k_{0}, \delta\right)}<1
$$

Now we separate the coefficients up to $k_{0}-1$ from the others and we set 


$$
a^{p}=\sum_{k=1}^{k_{0}-1} k\left|\xi_{k}^{p}\right|^{2} \quad \text { and } \quad b^{p}=\sum_{k=k_{0}}^{\infty} k\left|\xi_{k}\right|^{2}
$$

We know that

$$
\left\|e^{p}\right\|_{H^{1 / 2}\left(\Gamma^{*}\right)}^{2}=a^{p}+b^{p} .
$$

We also know that $a^{p} \geq 0$ and $a^{p+1}<a^{p}$, and moreover $b^{p}>0$ and $b^{p+1} \geq c\left(k_{0}, \delta\right)^{2} b^{p}$. When we iterate a sufficient number of times $a^{p}$ decreases faster than $b^{p}$. In fact

$$
0 \leq \frac{a^{p}}{b^{p}}=\frac{\sum_{k=1}^{k_{0}-1} k\left|\xi_{k}^{p}\right|^{2}}{\sum_{k=k_{0}}^{\infty} k\left|\xi_{k}^{p}\right|^{2}} \leq \frac{\sum_{k=1}^{k_{0}-1} k\left|\xi_{k}^{p}\right|^{2}}{k_{0}\left|\xi_{k_{0}}^{p}\right|^{2}}=\sum_{k=1}^{k_{0}-1} \frac{k}{k_{0}}\left|\eta_{k}^{p}\right|^{2} \rightarrow 0 \quad \text { when } p \rightarrow \infty
$$

because $k_{0}$ is not dependent on $p$. On the other hand we have

$$
b^{p+1} \geq c\left(k_{0}, \delta\right)^{2} b^{p}=\rho^{2} b^{p}+\epsilon b^{p},
$$

with $\epsilon=c\left(k_{0}, \delta\right)^{2}-\rho^{2}>0$. If $p$ is large enough, then $a^{p}<\epsilon b^{p}$ and we obtain

$$
\epsilon b^{p}>a^{p}-a^{p+1}>0
$$

and from this we prove

$$
\left\|e^{p+1}\right\|_{H^{1 / 2}\left(\Gamma^{*}\right)}^{2}=a^{p+1}+b^{p+1}>a^{p+1}+\rho^{2} b^{p}+a^{p}-a^{p+1} \geq \rho^{2} b^{p}+\rho^{2} a^{p}=\rho^{2}\left\|e^{p}\right\|_{H^{1 / 2}\left(\Gamma^{*}\right)}^{2},
$$

which is absurd, in view of the hypothesis.

To conclude this section, some remarks are in order. We first note that in practical algorithms, $f$ is only known on a finite number of Fourier modes. Therefore the algorithms converges to what can be viewed as approximate analytic continuation. The fact that convergence is slower when the number of modes increases reflects the fact that the exact analytic continuation problem is ill-posed in this case.

Concerning the convergence for more general geometries, the problem is essentially open. However, for boundaries that are small deformations of straight lines, a perturbation argument allows to prove convergence [4].

Finally, a numerical analysis of a fully discrete iterative method, incorporating in particular the effects of numerical quadrature for the evaluation of the integrals over $\Gamma$ and $\Gamma^{*}$, is possible for rectangular domains. Details of this analysis can be found in [4].

\section{NUMERICAL RESULTS}

In this section we outline the implementation of the fully discrete analytic continuation algorithm, and we present some numerical results that confirm the theoretical results. 


\subsection{Numerical Methods for the Solution of the Potential}

We solve the elliptic problems (22) using a second-order finite difference method. We define a grid on a domain that contains $\Omega *$. On the horizontal direction $[0,1]$ is divided in $N_{1}$ intervals and on the vertical direction $[-b, 0]$ is divided in $N_{2}$ intervals. The grid is extended above $x_{2}=$ 0 , to cover $\Omega^{*}$.

The results of the method are strongly dependent on the fictitious boundary $\Gamma^{*}$. On the one hand, $\Gamma^{*}$ must have a geometry as simple as possible to allow an easy numerical solution of problems (22). On the other hand, as we have seen in section $6, \Gamma^{*}$ must be as close to $\Gamma$ as possible, for the convergence not to be too slow and ill conditioned.

\subsubsection{The Boundary $\Gamma$}

The free boundary $\Gamma$ is discretized as a finite set of $N$ particles. Associated with each particle there is a potential (the value of the potential at the particle location). The integration formulas need some geometrical information about $\Gamma$. For this purpose we use cubic splines. Each coordinate of the parametrization of $\Gamma$ is represented by a cubic spline. The actual implementation uses spline routines from the Fishpack library. Periodicity was enforced by introducing extra knots on both sides of $\Gamma$.

\subsubsection{The Boundary $\Gamma^{*}$}

We construct above $\Gamma$ an artificial boundary $\Gamma^{*}$. Because we want to solve an elliptic problem on the domain $\Omega^{*}$ below $\Gamma^{*}$ using finite differences, we choose $\Gamma^{*}$ to be a polygonal line with its vertices lying on the uniform grid defined over $\Omega^{*}$. The artificial boundary $\Gamma^{*}$ is constructed by a general algorithm and is represented as a sequence of grid points that we name particles of $\Gamma^{*}$.

The construction of $\Gamma^{*}$ is dependent on the basic grid defined on the domain. If we refine this basic grid, $\Gamma^{*}$ is also refined. This refinement of $\Gamma^{*}$ affects not only the number of particles that describe this boundary, but also its shape. On the one hand, this effect is useful because a refinement of the base grid places $\Gamma^{*}$ closer to $\Gamma$. On the other hand, we cannot separate the effect of the refinement of the grid from the effect of the distance between $\Gamma$ and $\Gamma^{*}$. To separate these aspects of the discretization, it is possible to refine the finite difference grid and the number of the particles on $\Gamma^{*}$ without changing the shape of $\Gamma^{*}$. This refinement is controlled by an integer parameter $R$. After the construction of $\Gamma^{*}$, all horizontal and vertical intervals on the grid and all the segments of $\Gamma^{*}$ are divided in $R$ equal parts.

\subsubsection{The Solution of the Elliptic Problem}

We use an iterative Gauss-Seidel method with relaxation for the solution of the elliptic problem on the extended grid. The iteration starts with the values from the previous finite differenceintegral formula iteration. We iterate until the relative difference between two iterations is less than a certain parameter (in general we used either $10^{-6}$ or $0.5 \times 10^{-6}$ ), with a maximum number of iterations (usually 5000). The maximum number of iterations in the Gauss-Seidel iteration is only reached in the first iteration of the finite difference-integral formula cycle. In practice it is important to obtain an accurate solution of the elliptic problem to ensure that $\partial \phi / \partial n=0$ on $\Gamma_{b}$.

\subsubsection{Calculation of the Derivatives}

Once the potential $\phi$ is obtained, we need to calculate its derivatives on the particles of $\Gamma$. For the calculation of the velocity on a particle of $\Gamma$, we first identify the rectangular cell of the grid 
where the particle lies. Then each one of the derivatives is calculated in the corners of the cell, using a standard second-order formula. Finally, bilinear interpolation is used to approximate the values of the derivatives at the exact location of the particle.

\subsubsection{Calculation of the Integrals}

A direct implementation of the integral formula (23) gives rise to special problems on the calculation of the singular integrals on $\Gamma^{*}$. The boundary $\Gamma^{*}$ is a polygonal line with segments of different lengths and the approximation of Cauchy principal values in this situation is very delicate. We avoid these problems by noting that $\phi^{p}$ is a harmonic function on $\Omega * \backslash \Omega$, and therefore,

$$
\phi^{p}(x)=-2\left[\int_{\Gamma \cup \Gamma^{*}} \frac{\partial \tilde{\phi}^{p}}{\partial n}(y) G(x-y) d y+\int_{\Gamma \cup \Gamma^{*}} \tilde{\phi}^{p}(y) \frac{\partial G}{\partial n}(x-y) d y\right] .
$$

If we calculate the difference between $\phi^{p+1}$ and $\phi^{p}$, we obtain

$$
\phi^{p+1}(x)=\phi^{p}(x)-\frac{2 \pi}{\theta} \int_{\Gamma}\left(f(y)-\tilde{\phi}^{p}(y)\right) \frac{\partial G}{\partial n}(x-y) d y .
$$

This formula is of course equivalent to (23) at the continuous level. It is preferred to (23) at the discrete level because integration is computed only on $\Gamma$, which is a smooth line. Therefore its numerical integration by the standard trapezoidal rule yields spectral accuracy in the evaluation of the right hand side of (27).

If $\Gamma^{*}$ is too close to $\Gamma$, the value of $\partial G / \partial n$ tends to infinity. In practice this fact results in numerical instability. Therefore we cannot place $\Gamma^{*}$ as close to $\Gamma$ as would be desirable if we considered only the theoretical study of the model case.

Let us recall that $\theta$ denotes the value of the angle of $\Gamma^{*}$ at point $x$. In our construction, we view $\Gamma^{*}$ as a polygonal approximation of a smooth boundary lying on top of $\Gamma$. As a result, instead of taking for $\theta$ the local angles of this polygonal line, we always took $\theta=\pi$, which is the value for a smooth line.

\subsection{Numerical Results from the Analytic Continuation Algorithm}

For the numerical tests of the iterative algorithm, we considered a geometry with a horizontal bottom at depth $b=0.2$ and we set

$$
\Gamma=\left\{\left(x_{1}, 0.05 \cos \left(2 \pi x_{1}\right)\right): x_{1} \in[0,1]\right\}
$$

and

$$
\Gamma_{b}=\left\{\left(x_{1},-0.2\right): x_{1} \in[0,1]\right\}
$$

We name shape amplitude the parameter that multiplies the cosinus function $(0.05$, in this case).

As the given potential on $\Gamma$, we considered two functions with different regularities: 


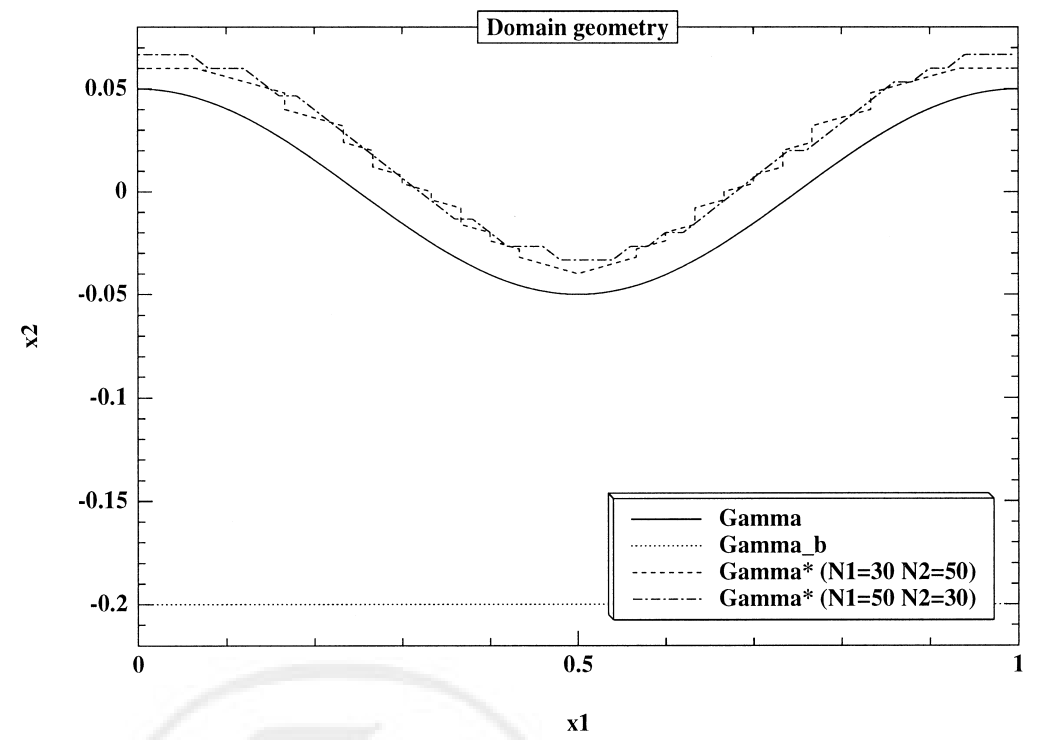

FIG. 8. Two domain geometries for depth 0.2 and shape amplitude 0.05 .

$$
\begin{gathered}
f_{1}\left(x_{1}, x_{2}\right)= \begin{cases}-\left(x_{1}+0.5\right)\left(x_{1}-0.5\right) & \text { if } x_{1}<0.5 \\
-\left(x_{1}-0.5\right)\left(x_{1}-1.5\right) & \text { if } x_{1} \geq 0.5\end{cases} \\
f_{2}\left(x_{1}, x_{2}\right)= \begin{cases}x_{1} & \text { if } x_{1}<0.5 \\
x_{1}-1 & \text { if } x_{1} \geq 0.5 .\end{cases}
\end{gathered}
$$

Function $f_{1}$ is only continuous and function $f_{2}$ is discontinuous.

In each case we ran 50 iterations of the analytic continuation algorithm. After each iteration the $L^{2}$ relative error of the potential on $\Gamma$ was calculated.

We recall the discretization parameters: $N$ is the number of particles on $\Gamma ; N_{1}$ is the base number of intervals in the $x_{1}$ direction, between 0 and $1 ; N_{2}$ is the base number of intervals in the $x_{2}$ direction, between $-b$ and $0 ; R$ is the refinement parameter for the creation of the finite difference subgrid. We remark that, for a given $\Gamma$, the artificial boundary $\Gamma^{*}$ is dependent only on $N_{1}$ and $N_{2}$.

In Fig. 8 we can see, together with $\Gamma$ and $\Gamma_{b}$, two artificial boundaries $\Gamma^{*}$, resulting from different discretization parameters. Figures 9 and 10 present the exact and numerical values of the potential on $\Gamma$.

The final result of the iterations is a harmonic function on $\Omega^{*}$, obtained by solving a Laplace equation with a boundary condition given on $\Gamma^{*}$ by the last iteration. Therefore the trace of this function on $\Gamma$ and its normal derivative on $\Gamma$ are as regular as the geometry of $\Gamma$ allows. If the data given on $\Gamma$ when we start the iterations, is not regular, the best we can expect is to obtain a good smooth approximation of this data.

When $\Gamma$ is a horizontal line and we run a fixed number of iterations, the theoretical results for the model case suggest that we would obtain better approximations for lower Fourier modes than for the higher ones. Therefore, the result of the application of a fixed number of iterations can be seen as a sort of sum of a truncated Fourier series of the initial function. Once again the theoretical results indicate that the number of Fourier modes well reproduced is dependent, on 


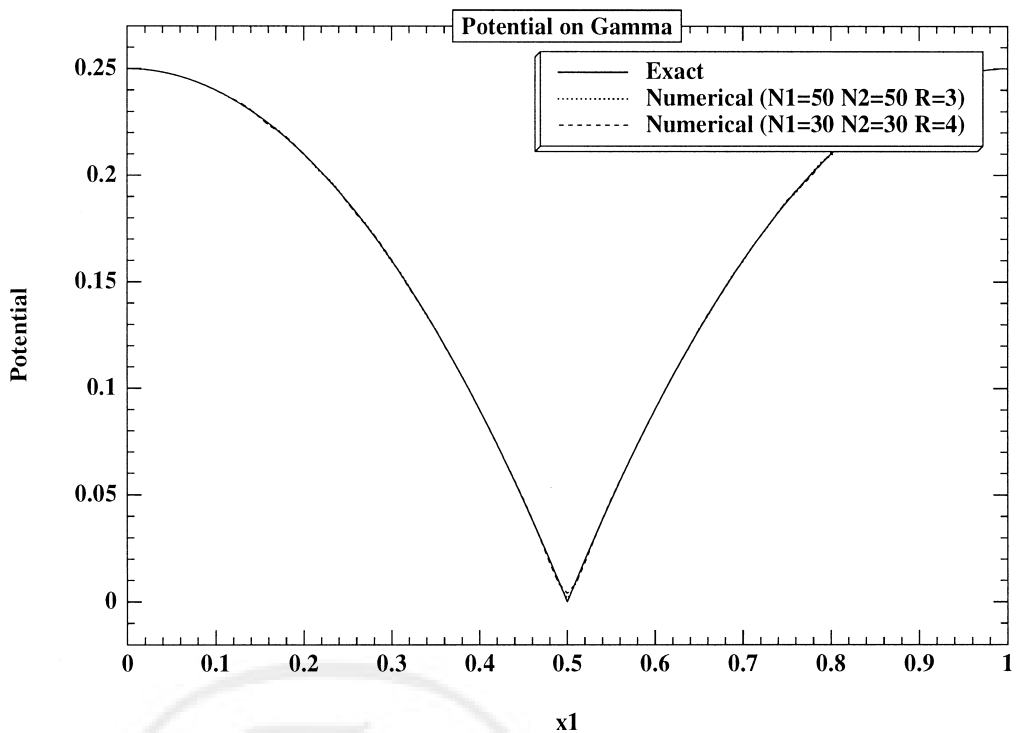

FIG. 9. Exact $\left(f_{1}, 28\right)$ and numerical values of the potential on $\Gamma$ with depth 0.2 , shape amplitude 0.05 , and $N=100$.

the one hand, on the discretization parameters and, on the other hand, on the distance between $\Gamma$ and $\Gamma^{*}$.

The numerical results presented in this section for non horizontal $\Gamma^{*}$ seem to reveal a similar effect, with the particularity that, for the discretization parameters we used, the dominant factor seems to be the distance between $\Gamma$ and $\Gamma^{*}$. When $N_{2}$ increases, the constructed $\Gamma^{*}$ is closer to

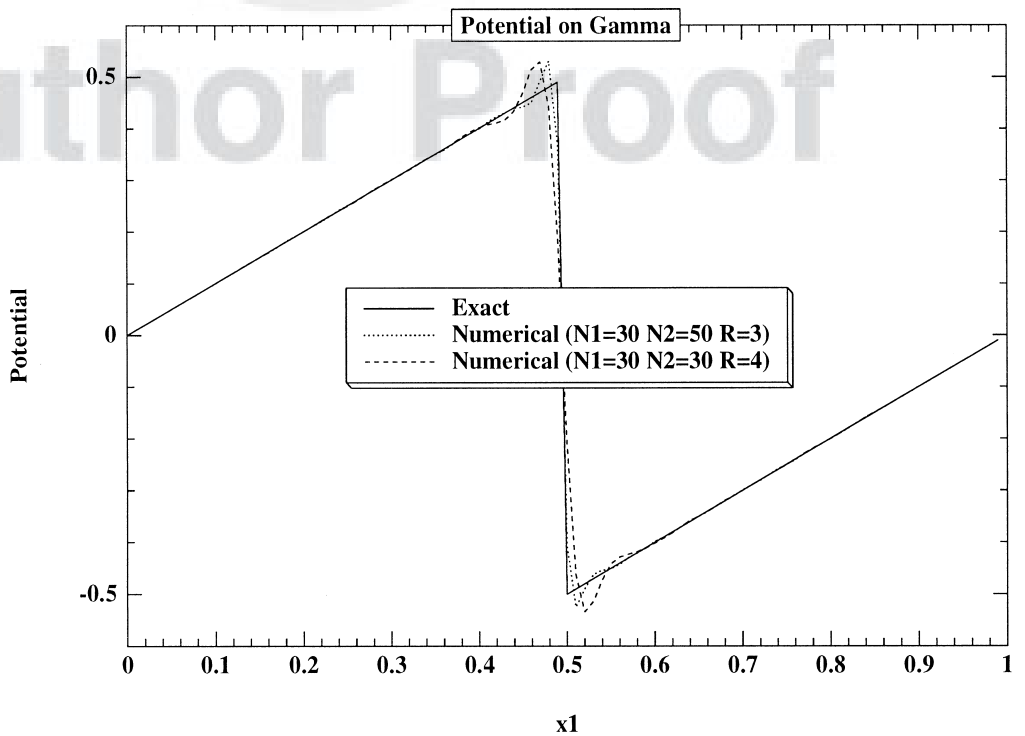

FIG. 10. Exact $\left(f_{2}, 29\right)$ and numerical values of the potential on $\Gamma$ with depth 0.2 , shape amplitude 0.05 , and $N=100$. 
$\Gamma$, and the results are better for less regular initial data. The refinement of the finite difference grid (obtained by increasing the parameter $R$ ) without changing the domain geometry does not seem to affect significantly the results of the algorithm. However, for regular data on $\Gamma$, the ratio between the sizes of the horizontal and vertical grids seems to have some importance. At this stage the number of points on $\Gamma$ seems to have little impact on the results. As could be expected in a Fourier type approximation, the results are better when the data on $\Gamma$ has more regularity. The overshooting we observe when the initial data is discontinuous seems to be a Gibbs' effect present when we calculate a partial sum of a Fourier series of a discontinuous function.

To summarize, the algorithm works well for more regular functions and, for less regular functions, captures the initial data function on a scale that is dependent on the distance between $\Gamma$ and $\Gamma^{*}$.

\section{CONCLUSION}

We have described and analyzed several iterative techniques based on simplified integral formulations that may be useful for the solution of elliptic problems in complex geometries. These methods are motivated by the computation of potentials in flow problems, either in exterior domains or with free boundaries. In the first case, the method is a variation of the Schwarz alternating domain decomposition method. Numerical analysis and experiments indicate that, as it could be expected, its rate of convergence is governed by the amount of overlapping between the subdomains. This method has been successfully applied to the numerical simulation of Navier-Stokes in exterior domains. In the second case, the method can be viewed as a numerical analytic continuation algorithm. Its convergence properties critically depend on a balance of regularity of the potential and of the distance between the free boundary and the fictitious boundary. The method gives good results in the numerical simulation of standing water waves but some improvement in the generation of the fictitious boundary seems still necessary to simulate overturning waves. The convergence is proved for simple geometries. The numerical analysis of the analytic continuation algorithm in the case of general geometries remains a challenging open problem for which a different approach seems to be necessary.

\section{APPENDIX: PROOFS OF LEMMAS}

Proof of Lemma 1. Let $w$ be the solution of (8) with $h \equiv 1$. Then, by the weak maximum principle $0<w(x)<1$ for any $x \in \Gamma_{2}$ and, because $\Gamma_{2}$ is compact, $0<\eta=\max _{x \in \Gamma_{2}} w(x)<$ 1. As $|h(x)| \leq M$ for all $x \in \Gamma_{1}$, we have

$$
-M w \leq v \leq M w \quad \text { on } \Gamma \cup \Gamma_{1}
$$

and, because $v$ and $w$ are harmonic in $\Omega_{1}$ we have

$$
-M w \leq v \leq M w \quad \text { in } \overline{\Omega_{1}},
$$

which gives $|v(x)| \leq M|w(x)|$ for all $x \in \overline{\Omega_{1}}$ and, in particular, $|u(x)| \leq \eta M$, for all $x \in \Gamma_{2}$.

To estimate the value of $\eta$, we consider the function 


$$
l(x)=\frac{\log \|x\|-\log R_{0}}{\log R_{1}-\log R_{0}}
$$

defined in $\Omega \cap B\left(0, R_{1}\right)$. The function $l$ is harmonic outside the origin and increases with $\|x\|$. Moreover, in $\Omega \cap B\left(0, R_{1}\right)$ we have $l \geq w$ because $l=1>w$ on $S\left(0, R_{1}\right)$ and $l \geq w=0$ on $\Gamma$. Now we have

$$
0 \leq \eta=\max _{\Gamma_{2}} w \leq \max _{\Gamma_{2}} l \leq \max _{S\left(0, R_{2}\right)} l=\frac{\log R_{2}-\log R_{0}}{\log R_{1}-\log R_{0}}
$$

Because $R_{0}$ and $R_{2}$ may be fixed, if $R_{1}$ is large enough, then $\eta$ is as small as necessary.

Proof of Lemma 2. We start by noting that the constant at infinity may be calculated from the Dirichlet data on $\Gamma_{2}$

$$
c=\int_{\Gamma_{2}} h \frac{\partial w}{\partial n} d \gamma
$$

where $w \in C^{2}(\Omega) \cap C^{1}(\bar{\Omega})$ is a function that depends only on $\Gamma_{2}$ [7].

We will prove that, for any $\delta>0$ and $x \in \Omega_{2}$

$$
|v(x)| \leq \max (M,|c|+\delta)
$$

which implies that

$$
|v(x)| \leq \max (M,|c|) \leq \max \left(M, M \int_{\Gamma_{2}}\left|\frac{\partial w}{\partial n}\right| d \gamma\right) \leq \theta M,
$$

with

$$
\theta=\max \left(1, \int_{\Gamma_{2}}\left|\frac{\partial w}{\partial n}\right| d \gamma\right)
$$

thus proving the lemma.

To see (30) we consider $x \in \Omega_{2}$ and $\delta>0$. From the behavior at infinity we know that there exists $R>\|x\|$ such that $|v(y)-c| \leq \delta$ for all $y \in S(0, R)$. If we consider $w_{1}$ and $w_{2}$ defined as the solutions of

$$
\begin{cases}\Delta w_{1}=0 & \text { in } \Omega_{2} \cap B(0, R) \\ w_{1}=h & \text { on } \Gamma_{2} \\ w_{1}=c-\delta & \text { on } S(0, R)\end{cases}
$$

and 


$$
\begin{cases}\Delta w_{2}=0 & \text { in } \Omega_{2} \cap B(0, R) \\ w_{2}=h & \text { on } \Gamma_{2} \\ w_{2}=c+\delta & \text { on } S(0, R)\end{cases}
$$

by the weak maximum principle we have

$$
w_{1} \leq v \leq w_{2} \quad \text { in } \quad \Omega_{2} \cap B(0, R),
$$

and therefore

$$
\min (M, c-\delta) \leq \min _{\Gamma_{2} \cup S(0, R)} w_{1} \leq v \leq \max _{\Gamma_{2} \cup S(0, R)} w_{2}=\max (M, c+\delta)
$$

and finally (30).

Proof of Lemma 3. Let $\Omega^{*}=B\left(0,2 R_{2}-R_{0}\right) \cap \Omega$ and let $x \in \Gamma_{2}$. Then $\partial \Omega^{*}=\Gamma \cup S(0$, $\left.2 R_{2}-R_{0}\right)$ and $d(x, \Gamma)<R_{2}-R_{0}<d\left(x, S\left(0,2 R_{2}-R_{0}\right)\right)$ and therefore $d\left(x, \partial \Omega^{*}\right)=d(x, \Gamma) \geq$ $d\left(\Gamma_{2}, \Gamma\right)$. We are now able to apply in $\Omega *$ the estimate for the derivatives of a harmonic function ([8]) and we get

$$
\|\nabla u(x)\| \leq \frac{2}{d\left(x, \Omega^{*}\right)} \sup _{\Omega^{*}}|u| \leq \frac{2}{d\left(\Gamma_{2}, \Gamma\right)} \sup _{\Omega^{*}}|u| .
$$

To prove that $\sup _{\Omega *}|u| \leq \eta^{*} M$ with

$$
\eta^{*}=\frac{\log \left(2 R_{2}-R_{0}\right)-\log R_{0}}{\log R_{1}-\log R_{0}},
$$

we only have to repeat the proof of Lemma 1, adapting the geometrical constraints.

AQ: 2

\section{References}

1. G.-H. Cottet, Particle-grid domain decomposition methods for the Navier-Stokes equations in exterior domains, C. Anderson and C. Greengard editors, Vortex dynamics and vortex methods, American Mathematical Society, Rhode Island, 1991, pp 103-117.

2. M. L. Ould-Salihi, G.-H. Cottet, and M. El Hamraoui, Blending finite-difference and vortex methods for incompressible flow computations, SIAM J Sci Comput 22(5) (2000), 1655-1674.

3. G.-H. Cottet and C. Albuquerque, Méthodes hybrides Particules-Grille pour des problèmes de frontière libre, Rapport intermédiaire DRET, 1992.

4. C. Albuquerque, Analytic continuation techniques and applications to free boundary problems, $\mathrm{PhD}$ Thesis, University of Lisbon, 2000.

5. C. Albuquerque and G.-H. Cottet, Analytic continuation techniques and applications to water waves, in preparation.

6. M.-N. Leroux, Méthode d'élements finis pour la resolution numérique de problèmes exterieurs en dimension 2, RAIRO 11 (1977), 27-60.

7. J. C. Nedelec, Notions sur les equations integrales de la physique-Theorie et approximation, Rapport Interne, CMAP.

8. T. Y. Hou, Numerical solutions to free boundary problems, Acta Numerica (1995), 335-415. 


\section{Suggested Readings}

1. C. Albuquerque, Métodes de decomposição de domínio para o problema exterior de Laplace, M.Sc. Thesis, University of Lisbon, 1991.

2. C. Albuquerque, Some domain-decomposition methods for the exterior Poisson equation, M. Marques et al., editors, Trends in applications of mathematics to mechanics, Longman, 1995, pp 299-310.

3. G. R. Baker, D. I. Meiron, and S. A. Orszag, Generalized vortex methods for free-surface flow problems, J Fluid Mech 123 (1982), 477-501.

4. J. T. Beale, T. Y. Hou, and J. Lowengrub, Convergence of a boundary integral method for water waves, SIAM J Numer Anal 33(5) (1996), 1797-1843.

5. X. Cai, H. P. Langtangen, B. F. Nielsen, and A. Tveito, A finite element method for fully nonlinear water waves, J Comput Phys 143 (1998), 544-568.

6. G.-H. Cottet, A particle grid superposition method for the Navier-Stokes equations, J Comput Phys 89 (1990), 301-318.

7. G.-H. Cottet and P. Koumoutsakos, Vortex methods-theory and practice, Cambridge University Press, 2000.

8. D. Gilbarg and S. Trudinger, Elliptic partial differential equations of second order, Springer Verlag, New York, 1983.

9. T. Y. Hou, Numerical study of free interface problems using boundary integral methods, Documenta Mathematica, Extra Volume ICM, III (1998), 601-610.

10. M. S. Longuet-Higgins and E. D. Cokelet, The deformation of steep surface waves on water I, Proc R Soc London 350 (1976), 1-26.

11. W. L. Wendland, Boundary element methods for elliptic problems. Mathematical theory of finite and boundary element methods, Birkhauser Verlag, 1990. 